Pure and Applied Mathematics Quarterly

Volume 3, Number 2

(Special Issue: In honor of

Leon Simon, Part 1 of 2$)$

$451-480,2007$

\title{
Vector-valued Singular Integral Operators with Non-smooth Kernels and Related Multilinear Commutators*
}

\author{
Hui-Xia Mo and Shan-Zhen Lu
}

\begin{abstract}
Let $(\mathbb{X}, d, \mu)$ be a space of homogeneous type. First, the authors introduced the notation of vector-valued singular integral operator $T$ with non-smooth kernel on $\mathbb{X}$, and studied the boundedness of $T$ and its maximal truncated operator $T_{*}$. Then, the authors gave the definition of multilinear commutator generated by $T$ and $B M O$ functions or by $T$ and Lipschitz functions, and discussed the boundedness of these multilinear commutators.
\end{abstract}

\section{INTRODUCTION}

Let $\mathbb{X}$ be a topological space equipped with a distance $d$ and a non-negative Borel regular measure $\mu$. ( $\mathbb{X}, d, \mu)$ is called to be a space of homogeneous type, if the doubling condition

$$
\mu(B(x, 2 r)) \leq c \mu(B(x, r))<\infty
$$

holds for some $c \geq 1$ uniformly and for all $x \in \mathbb{X}$ and $r>0$, where $B(x, r)=$ $\{y \in \mathbb{X}: d(x, y)<r\}$. A more general definition for this space can be found in [1], [2] and [3]. Note that the doubling property implies the following strong homogeneity property

$$
\mu(B(x, \lambda r)) \leq c \lambda^{n} \mu(B(x, r)),
$$

for some $c, n$ uniformly and for all $\lambda \geq 1$. The parameter $n$ is a measure of dimension of the space. There also exist $c>0$ and $N(0 \leq N \leq n)$ so that

$$
\mu(B(y, r)) \leq c\left(1+\frac{d(x, y)}{r}\right)^{N} \mu(B(x, r)),
$$

uniformly for all $x, y \in \mathbb{X}$ and $r>0$. In fact, the property (1.3) with $N=n$ is a direct consequence of triangle inequality of the distance $d$ and the strong

Received December 18, 2005.

*Supported by the NNSF and SEDF of China(20040027001) 
homogeneity property. But in general $N$ can be smaller. In the cases of Euclidean spaces $\mathbb{R}^{n}$ and Lie groups of polynomial growth, $N$ can be chosen to be 0 .

Throughout this paper we shall work on space of homogeneous type $(\mathbb{X}, d, \mu)$ with $\mu(\mathbb{X})=\infty$, and $C$ denotes different constant in different line.

Let $T$ be a bounded linear operator on $L^{2}(\mathbb{X})$ with an associated kernel $K(x, y)$ in the sense that

$$
T f(x)=\int_{\mathbb{X}} K(x, y) f(y) d \mu(y),
$$

where $K(x, y)$ is a measurable function, and the above formula holds for each continuous function $f$ with compact support, and for almost all $x$ not in the support of $f$.

One important result of Calderón-Zymund operator theory is the well known Hörmander integral condition on the kernel $K(x, y)$, which is a sufficient condition for the operator $T$ to be of weak type $(1,1)$ (see [4]). However, there are numerous examples of operators which do not satisfy Hörmander integral condition, and certain classes of such operators can be proved to be of weak type $(1,1)$. See, for example [5], [6] and [7]. A natural question is whether one can weaken the Hörmander integral condition and still conclude that $T$ is an operator of weak type $(1,1)$. In 1999, Duong and McIntosh [8] gave an affirmative answer to this question and introduced the notation of singular integral operator with nonsmooth kernel on space of homogeneous type $\mathbb{X}$.

On the other hand, Duong and Yan [9] studied the boundedness of commutator generated by the singular integral operator with non-smooth kernel and $B M O$ function. In the paper, we will introduced the notation of vector-valued singular integral operator with non-smooth kernel on space of homogeneous type and extend the results in [8] and [9] to the vector-valued situation.

Given a Banach space $H$, we shall denote by $L_{H}^{p}(\mathbb{X})$ or simply by $L_{H}^{p}$ the Bochner-Lebesgue spaces of $H$-valued strongly measurable functions $f$ such that

$$
\|f\|_{L_{H}^{p}(\mathbb{X})}=\left(\int_{\mathbb{X}}\|f(x)\|_{H}^{p} d \mu(x)\right)^{1 / p}<\infty, \quad \text { for all } 1 \leq p<\infty,
$$

in which $\|f(x)\|_{H}$ denotes the norm of $f$ in $H$. When $H$ is the scalar filed we simply write $L^{p}(\mathbb{X})=L_{H}^{p}(\mathbb{X})$. For $p=\infty$, we write $L_{H}^{\infty}(\mathbb{X})$ for the space of all of the functions $f$ such that $\|f\|_{L_{H}^{\infty}(\mathbb{X})}=\operatorname{ess} \sup \|f\|_{H}<\infty$, and $L_{c, H}^{\infty}(\mathbb{X})$ for the space of all compactly supported members of $L_{H}^{\infty}(\mathbb{X})$.

Let $E$ and $F$ be a couple of Banach spaces, we denote by $\mathcal{L}(E, F)$ all of the bounded linear operators from $E$ to $F$. And set $\mathcal{L}(E)=\mathcal{L}(E, E)$ for short.

Definition 1.1. Let $H$ be a Banach space. A family of operators $\left\{A_{t}: t>0\right\} \subset$ $\mathcal{L}(H)$ is said to be an "approximation to identity" on $H$ if, for every $t>0, A_{t}$ is 
represented by the kernel $a_{t}(x, y): \mathbb{X} \times \mathbb{X} \longmapsto \mathcal{L}(H)$, in the following sense: for every $f \in L_{H}^{p}(\mathbb{X}), p \geq 1$,

$$
A_{t} f(x)=\int_{\mathbb{X}} a_{t}(x, y) f(y) d \mu(y)
$$

and the following condition holds:

$$
\left\|a_{t}(x, y)\right\|_{\mathcal{L}(H)} \leq h_{t}(x, y),
$$

for all $x, y \in \mathbb{X}$. Where $h_{t}(x, y)$ is a function satisfying

$$
h_{t}(x, y)=\frac{s\left(d(x, y)^{m} t^{-1}\right)}{\mu\left(B\left(x, t^{1 / m}\right)\right)}
$$

in which $m$ is a positive constant and $s$ is a positive, bounded, decreasing function satisfying

$$
\lim _{r \rightarrow \infty} r^{n+N+\tau} s\left(r^{m}\right)=0
$$

for some $\tau>0$, here $N$ is the power which appeared in property (1.3) and $n$ is the "dimension" entering the strong homogeneity property.

Note that there exists a constant $C>0$ such that

$$
C^{-1} \leq \int_{\mathbb{X}} h_{t}(x, y) d \mu(x) \leq C,
$$

uniformly for $y \in \mathbb{X}$ and $t>0$.

Moreover, for a locally integrable $H$-valued function $f$, the $H$-valued sharp maximal function associated with the "approximation to identity" $\left\{A_{t}: t>0\right\}$ is defined by

$$
M_{A}^{\#}\left(\|f\|_{H}\right)(x)=\sup _{x \in B} \frac{1}{\mu(B)} \int_{B}\left\|f(y)-A_{t_{B}} f(y)\right\|_{H} d \mu(y),
$$

where $t_{B}=r_{B}^{m}$ with $r_{B}$ is the radius of the ball $B$.

In the scalar case, Duong and McIntosh [8] introduced the notation of "approximation to identity" and constructed $\tilde{a}_{t}(x, y)$ which satisfies our conditions. In addition, Martell [10] gave the definition of $M_{A}^{\#}$ and get an analogy of the Fefferman-Stein estimate for the classical sharp maximal function.

After some preliminaries, in Section 2, we will introduce the definition of vectorvalued singular integral operator with non-smooth kernel and study the boundedness of $T$. In Section 3, we will study the maximal truncated operator $T_{*}$. In section 4 , the boundedness of multilinear commutator generated by $T$ and $B M O$ functions or by $T$ and Lipschitz functions will be discussed. 


\section{VECTOR-VALUED SINGULAR INTEGRAL OPERATOR WITH NON-SMOOTH KERNEL}

Definition 2.1. Let $E$ and $F$ be a couple of Banach spaces. A linear operator $T$, mapping $E$-valued functions into $F$-valued functions, is said to be a vectorvalued singular integral operator with non-smooth kernel $K(x, y): \mathbb{X} \times \mathbb{X} \longmapsto$ $\mathcal{L}(E, F)$, if $T$ is bounded from $L_{E}^{2}(\mathbb{X})$ to $L_{F}^{2}(\mathbb{X})$ and for any $f \in L_{c, E}^{\infty}(\mathbb{X})$,

$$
T f(x)=\int_{\mathbb{X}} K(x, y) f(y) d \mu(y) \text { for a.e } x \notin \operatorname{supp} f .
$$

Where $K$ satisfies the following conditions:

(i) There exists an "approximation to identity" $\left\{A_{t}: t>0\right\}$ on $E$ such that $T A_{t}$ has an associated kernel $k_{t}(x, y)$, and there exist constants $c_{1}, C>0$, so that

$$
\int_{d(x, y) \geq c_{1} t^{1 / m}}\left\|K(x, y)-k_{t}(x, y)\right\|_{\mathcal{L}(E, F)} d \mu(x) \leq C, \quad \text { for all } y \in \mathbb{X} .
$$

(ii) There exists an "approximation to identity" $\left\{A_{t}^{\prime}: t>0\right\}$ on $F$ such that $A_{t}^{\prime} T$ has a kernel $K_{t}(x, y)$, and there exist constants $c_{1}^{\prime}, C>0$, so that

$$
\int_{d(x, y) \geq c_{1}^{\prime} t^{1 / m}}\left\|K(x, y)-K_{t}(x, y)\right\|_{\mathcal{L}(E, F)} d \mu(y) \leq C, \quad \text { for all } x \in \mathbb{X} .
$$

(In fact, without loss of generality in what follows we will assume that $c_{1}=c_{1}^{\prime}=$ $1)$.

Let us show that in certain cases the vector-valued Hörmander integral condition implies (i) or (ii) in Definition 2.1.

Proposition 2.2. Assume that $T$ is a linear operator, mapping $E$-valued functions into $F$-valued functions, with an associated kernel $K(x, y): \mathbb{X} \times \mathbb{X} \longmapsto$ $\mathcal{L}(E, F)$ which satisfies the vector-value Hörmander integral condition, that is there exist constants $C>0$ and $\delta>1$, so that

$$
\int_{d(x, y) \geq \delta d(y, z)}\|K(x, y)-K(x, z)\|_{\mathcal{L}(E, F)} d \mu(x) \leq C,
$$

for all $y, z \in \mathbb{X}$.

Let $A_{t}$ be an "approximations to the identity" on $E$ which are represented by the kernel $a_{t}(x, y)$ satisfying the following additional properties:

(i) $a_{t}(x, y)=\theta_{E}$, when $d(x, y) \geq c_{0} t^{1 / m}$,

(ii) $\int_{\mathbb{X}} a_{t}(x, y) d \mu(x)=I_{E}$, for all $y \in \mathbb{X}, t>0$,

in which $\theta_{E}$ and $I_{E}$ denote the zero element and unit element in $\mathcal{L}(E)$, respectively. 
Then the kernel $k_{t}(x, y)$ of $T A_{t}$ satisfies the condition (i) of Definition 2.1, that is there exist constants $\beta, C>0$ such that

$$
\int_{d(x, y) \geq \beta t^{1 / m}}\left\|K(x, y)-k_{t}(x, y)\right\|_{\mathcal{L}(E, F)} d \mu(x) \leq C,
$$

for all $y \in \mathbb{X}$.

To prove Proposition 2.2 we follow the idea of [8].

Proof: Choose $\delta>1$ and let $\beta=c_{0} \delta$, where $c_{0}$ is the constant so that $a_{t}(x, y)=$ $\theta_{E}$, when $d(x, y) \geq c_{0} t^{1 / m}$. Then for all $y \in \mathbb{X}$,

$$
\begin{aligned}
& \int_{d(x, y) \geq \beta t^{1 / m}}\left\|K(x, y)-k_{t}(x, y)\right\|_{\mathcal{L}(E, F)} d \mu(x) \\
&= \int_{d(x, y) \geq \beta t^{1 / m}}\left\|K(x, y) I_{E}-\int_{\mathbb{X}} K(x, z) a_{t}(z, y) d \mu(z)\right\|_{\mathcal{L}(E, F)} d \mu(x) \\
&= \int_{d(x, y) \geq \beta t^{1 / m}} \| K(x, y) \int_{d(z, y) \leq c_{0} t^{1 / m}} a_{t}(z, y) d \mu(z) \\
&-\int_{\mathbb{X}} K(x, z) a_{t}(z, y) d \mu(z) \|_{\mathcal{L}(E, F)} d \mu(x) \\
&= \int_{d(x, y) \geq \beta t^{1 / m}}\left\|\int_{d(z, y) \leq c_{0} t^{1 / m}}(K(x, y)-K(x, z)) a_{t}(z, y) d \mu(z)\right\|_{\mathcal{L}(E, F)} d \mu(x) \\
& \leq \sup _{d(z, y) \leq c_{0} t^{1 / m}}\left(\int_{d(x, y) \geq \beta t^{1 / m}}\|K(x, y)-K(x, z)\|_{\mathcal{L}(E, F)} d \mu(x)\right) \\
& \quad \times\left(\int_{d(z, y) \leq c_{0} t^{1 / m}}\left\|a_{t}(z, y)\right\|_{\mathcal{L}(E)} d \mu(z)\right) \\
& \leq \sup _{d(x, y) \leq c_{0} t^{1 / m}}\left(\int_{d(x, y) \geq c_{0} \delta t^{1 / m}}\|K(x, y)-K(x, z)\|_{\mathcal{L}(E, F)} d \mu(x)\right) \\
& \quad \times\left(\int_{d(z, y) \leq c_{0} t^{1 / m}} h_{t}(z, y) d \mu(z)\right) \leq C .
\end{aligned}
$$

In the last inequality we use (1.7).

Remark 1. When the kernels of "approximations to identity" $\left\{A_{t}^{\prime}: t>0\right\}$ on $F$ satisfy the similar condition as (i) and (ii) in Proposition 2.2, we can show that if $K(x, y) \in \mathcal{L}(E, F)$ satisfies the following vector-valued Hörmander integral condition that is there exist $C>0$ and $\delta>1$, so that

$$
\int_{d(x, y) \geq \delta d\left(x, x^{\prime}\right)}\left\|K(x, y)-K\left(x^{\prime}, y\right)\right\|_{\mathcal{L}(E, F)} d \mu(y) \leq C,
$$

for all $x, x^{\prime} \in \mathbb{X}$, then condition (ii) in Definition 2.1 also holds. 
Theorem 2.3. Let $T$, mapping $E$-valued functions into $F$-valued functions, be a vector-valued singular integral operator with non-smooth kernel, then there exists a constant $C>0$ such that

(i) $\|T f\|_{L_{F}^{p}(\mathbb{X})} \leq C\|f\|_{L_{E}^{p}(\mathbb{X})}$, for $1<p<\infty$,

(ii) $\mu\left(\left\{x \in \mathbb{X}:\|T f(x)\|_{F}>\lambda\right\}\right) \leq C\|f\|_{L_{E}^{1}(\mathbb{X})}$, for all $\lambda>0$,

(iii) $\left\|M_{A^{\prime}}^{\#}\left(\|T f\|_{F}\right)\right\|_{L^{\infty}(\mathbb{X})} \leq C\|f\|_{L_{E}^{\infty}(\mathbb{X})}$, for $f \in L_{E}^{2}(\mathbb{X}) \cap L_{E}^{\infty}(\mathbb{X})$.

Remark 2. In the scalar field, when $\mathbb{X}=\mathbb{R}^{n}$ is Euclidean space, (iii) in Theorem 2.3 implies that $T$ is bounded from $L^{\infty}\left(\mathbb{R}^{n}\right) \cap L^{2}\left(\mathbb{R}^{n}\right)$ to $B M O_{A^{\prime}}$. Where $B M O_{A^{\prime}}$ is the $B M O$ type space associated with an "approximation to identity" $\left\{A_{t}^{\prime}: t>\right.$ $0\}$. (The definition of $B M O_{A^{\prime}}$ can be seen in [11]).

Given a singular integral operator $T$, mapping $E$-valued functions into $F$-valued functions, with non-smooth kernel $K(x, y)$, then a new operator $\tilde{T}$ mapping $l^{q}(E)$-valued functions into $l^{q}(F)$-valued functions(where $q$ is fixed and $1<q<$ $\infty)$ can be defined by

$$
\tilde{T}\left(f_{1}, f_{2}, \cdots, f_{j}, \cdots\right)=\left(T f_{1}, T f_{2}, \cdots, T f_{j}, \cdots\right) .
$$

Thus the kernel associated with $\tilde{T}$ is $\tilde{K}=K \otimes I_{l^{q}(E)}$, and the two "approximation to identities" corresponding to Definition 2.1 are $\left\{\tilde{A}_{t}=A_{t} \otimes I_{l_{E}^{q}}: t>0\right\}$ and $\left\{\tilde{A}_{t}^{\prime}=A_{t}^{\prime} \otimes I_{l_{F}^{q}}: t>0\right\}$, where $I_{l_{E}^{q}}$ and $I_{l_{F}^{q}}$ denote the identity operators on $l_{E}^{q}$ and $l_{F}^{q}$ respectively. It is easy to check that the kernel of $\tilde{T}$ satisfy the condition (i) and (ii) in Definition 2.1, so we have the following corollary.

Corollary 2.4. If $T$, mapping $E$-valued functions into $F$-valued functions, is a vector-valued singular integral operator with non-smooth kernel, then for all $1<p, q<\infty$ and $\lambda>0$, there exists a constant $C>0$ such that

(i) $\left\|\left(\sum_{j}\left\|T f_{j}\right\|_{F}^{q}\right)^{1 / q}\right\|_{L^{p}(\mathbb{X})} \leq C\left\|\left(\sum_{j}\left\|f_{j}\right\|_{E}^{q}\right)^{1 / q}\right\|_{L^{p}(\mathbb{X})}$,

(ii) $\mu\left(\left\{x \in \mathbb{X}:\left(\sum_{j}\left\|T f_{j}(x)\right\|_{F}^{q}\right)^{1 / q}>\lambda\right\}\right) \leq C \lambda^{-1}\left\|\left(\sum_{j}\left\|f_{j}\right\|_{E}^{q}\right)^{1 / q}\right\|_{L^{1}(\mathbb{X})}$,

(iii) $\left\|M_{A^{\prime}}^{\#}\left(\sum_{j}\left\|T f_{j}\right\|_{F}^{q}\right)^{1 / q}\right\|_{L^{\infty}(\mathbb{X})} \leq C\left\|\left(\sum_{j}\left\|f_{j}\right\|_{E}^{q}\right)^{1 / q}\right\|_{L^{\infty}(\mathbb{X})}$, for all $f_{j} \in L_{E}^{2}(\mathbb{X}) \cap$ $L_{E}^{\infty}(\mathbb{X})$.

To prove Theorem 2.3 we need the following Lemmas.

Lemma 2.5.([12]) Given function $h_{t}(x, z)$ which satisfies (1.5) and $\nu>0$, then there exist positive constants $C$ and $\eta$ such that

$$
\sup _{z \in B(y, r)} h_{t}(x, z) \leq C \inf _{z \in B(y, r)} h_{\eta t}(x, z),
$$


uniformly for $x, y \in \mathbb{X}$ and $r, t>0$ with $r^{m} \leq \nu t$.

Lemma 2.6. For every $f \in L_{H}^{p}(\mathbb{X}), 1 \leq p<\infty$ and $t>0$, we have

(i) $\int_{\mathbb{X}} h_{t}(x, y)\|f(y)\|_{H} d \mu(y) \leq C M\left(\|f\|_{H}\right)(x)$,

(ii) $\int_{\mathbb{X}} h_{t}(x, y)\|f(x)\|_{H} d \mu(x) \leq C M\left(\|f\|_{H}\right)(y)$.

In fact we need only to prove (i), for (ii) it is similar to.

Take $B\left(x, r_{B}\right)$ with $r_{B}=t^{1 / m}$, then using (1.5),

$$
\begin{aligned}
& \int_{\mathbb{X}} h_{t}(x, y)\|f(y)\|_{H} d \mu(y) \\
& \leq \int_{d(x, y)<2 r_{B}} \frac{s\left(d(x, y)^{m} t^{-1}\right)}{\mu\left(B\left(x, r_{B}\right)\right)}\|f(y)\|_{H} d \mu(y) \\
& \quad+\sum_{k=1}^{\infty} \int_{2^{k} r_{B} \leq d(x, y)<2^{k+1} r_{B}} \frac{s\left(d(x, y)^{m} t_{B}^{-1}\right)}{\mu\left(B\left(x, r_{B}\right)\right)}\|f(y)\|_{H} d \mu(y) \\
& :=I+I I .
\end{aligned}
$$

Since $s(x, y)$ is a positive, bounded, decreasing function, thus by the doubling condition (1.1), we have

$$
I \leq C s(0) \frac{1}{\mu\left(B\left(x, 2 r_{B}\right)\right)} \int_{d(x, y)<2 r_{B}}\|f(y)\|_{H} d \mu(y) \leq M\left(\|f\|_{H}\right)(x) .
$$

Moreover, when $2^{k} r_{B} \leq d(x, y)<2^{k+1} r_{B}$ by the strong homogeneity property $(1.2)$

$$
\frac{s\left(d(x, y)^{m} t_{B}^{-1}\right)}{\mu\left(B\left(x, r_{B}\right)\right)} \leq \frac{C\left(2^{k+1}\right)^{n} s\left(2^{k m}\right)}{\mu\left(B\left(x, 2^{k+1} r_{B}\right)\right)} .
$$

Thus using (1.6), we have

$$
\begin{aligned}
& I I \leq \sum_{k=1}^{\infty} C \int_{2^{k} r_{B} \leq d(x, y)<2^{k+1} r_{B}} \frac{\left(2^{k+1}\right)^{n} s\left(2^{k m}\right)}{\mu\left(B\left(x, 2^{k+1} r_{B}\right)\right)}\|f(y)\|_{F} d \mu(y) \\
& \leq \sum_{k=1}^{\infty} C 2^{k n} s\left(2^{k m}\right) \frac{1}{\mu\left(B\left(x, 2^{k+1} r_{B}\right)\right)} \int_{d(x, y)<2^{k+1} r_{B}}\|f(y)\|_{H} d \mu(z) \\
& \leq \sum_{k=1}^{\infty} C 2^{-k(N+\tau)} 2^{k(n+N+\tau)} s\left(2^{k m}\right) M\left(\|f\|_{H}\right)(x) \\
& \leq C M\left(\|f\|_{H}\right)(x) .
\end{aligned}
$$

Combining the estimate of $I$ with $I I$, we prove Lemma 2.6.

Lemma 2.7.([1]) (Calderón-Zygmund decomposition on homogeneous space) 
Let $f \geq 0, f \in L^{1}(\mathbb{X})$ with compact support and $\lambda>0$. Then there exists a family of balls $\left\{B_{i}\right\}$ such that:

(i) $f \leq C \lambda$ for $\mu$ a.e. $x \in \mathbb{X} \backslash \bigcup_{i} B_{i}$,

(ii) $\frac{1}{\mu\left(B_{i}\right)} \int_{B_{i}} f(y) d \mu(y) \leq C \lambda$,

(iii) $\sum_{i} \mu\left(B_{i}\right) \leq \frac{C}{\lambda} \int_{\mathbb{X}} f(y) d \mu(y)$,

(iv) There exists an integer $M \geq 1$, independent of $f$ and $\lambda$, such that every point in $\mathbb{X}$ belongs to at most $M$ of these balls.

Remark 3. The following properties are contained in the proof of the previous results:

(i) There exists some constant $C_{\mathbb{X}}$, which only depends on the space $\mathbb{X}$, such that

$$
\Omega_{\lambda}=\left\{x \in \mathbb{X}: M f(x)>\lambda C_{\mathbb{X}}\right\}=\bigcup_{i} B_{i},
$$

here $M f$ denotes the Hardy-Littlewoood maximal function of $f$.

(ii) There exists $\varepsilon_{0}>1$ independent of $f$ and $\lambda$ such that $\left(\varepsilon_{0} B_{i}\right) \backslash \Omega_{\lambda} \neq \varnothing$

Now, Let us turn to prove Theorem 2.3.

For $1<p<2$, it is a straightforward generalization of Theorem 1 in [8]. But here we need the Calderón-Zygmund decomposition for $\|f\|_{E}$ as follows.

For $\|f\|_{E}$, by Lemma 2.7 and Remark 3 , there exists a collection of balls $\left\{B_{i}\right\}$ such that

$$
\Omega_{\lambda}=\left\{x \in \mathbb{X}: M\left(\|f\|_{E}\right)(x)>\lambda C_{\mathbb{X}}\right\}=\bigcup_{i} B_{i} .
$$

As in [10], we decompose $f$ as $f=g+b=g+\sum_{i} b_{i}$, where

$$
\begin{aligned}
& g(x)=f(x) \chi_{\mathbb{X} \backslash \bigcup_{i} B_{i}}+\sum_{i}\left(\frac{1}{\mu\left(B_{i}\right)} \int_{B_{i}} f(y) \rho_{i}(y) d \mu(y)\right) \chi_{B_{i}(x)}, \\
& b_{i}(x)=f(x) \rho_{i}(x)-\left(\frac{1}{\mu\left(B_{i}\right)} \int_{B_{i}} f(y) \rho_{i}(y) d \mu(y)\right) \chi_{B_{i}(x)},
\end{aligned}
$$

and

$$
\rho_{i}(x)=\frac{\chi_{B_{i}}(x)}{\sum_{j} \chi_{B_{j}}(x)} \chi_{\bigcup j B_{j}}(x) .
$$

So by (iv) in Lemma 2.7 and Remark 3 , we have the following conclusions:
(a) If $x \in B_{i}$, then $M^{-1} \leq \rho_{i}(x) \leq 1$. Moreover, $\sum_{i} \rho_{i}(x)=\chi_{\cup_{j} B_{j}}(x)$.
(b) $\|g(x)\|_{E} \leq C \lambda$ for $\mu$ a.e $x \in \mathbb{X}$.
(c) $\operatorname{supp} b_{i} \subset B_{i}$ and $\frac{1}{\mu\left(B_{i}\right)} \int_{B_{i}}\left\|b_{i}(x)\right\|_{E} d \mu(x) \leq C \lambda$. 
Thus,

$$
\begin{aligned}
& \mu\left(\left\{x \in \mathbb{X}:\|T f(x)\|_{F}>\lambda\right\}\right) \\
& \leq \mu\left(\left\{x \in \mathbb{X}:\|T g(x)\|_{F}>\lambda / 2\right\}\right)+\mu\left(\left\{x \in \mathbb{X}:\|T b(x)\|_{F}>\lambda / 2\right\}\right) .
\end{aligned}
$$

For the "good" part, notice that $T$ is bounded from $L_{E}^{2}(\mathbb{X})$ to $L_{F}^{2}(\mathbb{X})$, so by the Chebyshev inequality and (b), we have

$$
\begin{aligned}
& \mu\left(\left\{x \in \mathbb{X}:\|T g(x)\|_{F}>\lambda / 2\right\}\right) \\
& \leq \frac{C}{\lambda^{2}} \int_{\mathbb{X}}\|T g(x)\|_{F}^{2} d \mu(x) \\
& \leq \frac{C}{\lambda^{2}} \int_{\mathbb{X}}\|g(x)\|_{E}^{2} d \mu(x) \\
& \leq \frac{C}{\lambda} \int_{\mathbb{X}}\|g(x)\|_{E} d \mu(x) \\
& \leq \frac{C}{\lambda}\left(\int_{\mathbb{X} \backslash \Omega_{\lambda}}\|g(x)\|_{E} d \mu(x)+\int_{\Omega_{\lambda}}\|g(x)\|_{E} d \mu(x)\right) \\
& \leq \frac{C}{\lambda}\left(\|f\|_{L_{E}^{1}(\mathbb{X})}+\int_{\Omega_{\lambda}}\left\|\sum_{i}\left(\frac{1}{\mu\left(B_{i}\right)} \int_{B_{i}} f(y) \rho_{i}(y) d \mu(y)\right) \chi_{B_{i}(x)}\right\|_{E} d \mu(x)\right) \\
& \leq \frac{C}{\lambda}\left(\|f\|_{L_{E}^{1}(\mathbb{X})}+\int_{\Omega_{\lambda}} \sum_{i}\left(\frac{1}{\mu\left(B_{i}\right)} \int_{B_{i}}\left\|f(y) \rho_{i}(y)\right\|_{E} d \mu(y)\right) \chi_{B_{i}(x)} d \mu(x)\right) \\
& \leq \frac{C}{\lambda}\left(\|f\|_{L_{E}^{1}(\mathbb{X})}+\sum_{i} \int_{B_{i}}\|f(y)\|_{E} d \mu(y)\right) \\
& \leq \frac{C}{\lambda}\|f\|_{L_{E}^{1}(\mathbb{X})} .
\end{aligned}
$$

Let us now estimate the "bad" part. Set $t_{i}=r_{B_{i}}^{m}$ and write

$$
T b_{i}=T A_{t_{i}} b_{i}(x)+\left(T-T A_{t_{i}}\right) b_{i}(x) .
$$

Then

$$
\begin{aligned}
& \left\|A_{t_{i}} b_{i}(x)\right\|_{E} \leq \int_{\mathbb{X}}\left\|a_{t_{i}}(x, y)\right\|_{\mathcal{L}(E)}\left\|b_{i}(y)\right\|_{E} d \mu(y) \\
& \leq \int_{\mathbb{X}} h_{t_{i}}(x, y)\left\|b_{i}(y)\right\|_{E} d \mu(y) \\
& \leq \sup _{y \in B_{i}} h_{t_{i}}(x, y) \int_{B_{i}}\left\|b_{i}(y)\right\|_{E} d \mu(y) \\
& \leq C \lambda \mu\left(B_{i}\right) \inf _{y \in B_{i}} h_{\eta t_{i}}(x, y) \\
& \leq C \lambda \int_{\mathbb{X}} h_{\eta t_{i}}(x, y) \chi_{B_{i}}(y) d \mu(y) .
\end{aligned}
$$

Take $\varphi \in L^{2}(\mathbb{X})$, then by (2.3), Lemma 2.6 and using the idea of [8], 


$$
\begin{aligned}
& |<| \varphi\left|,\left\|A_{t_{i}} b_{i}\right\|_{E}>\right| \\
& \leq C \lambda \int_{\mathbb{X}} \int_{\mathbb{X}}|\varphi(x)| h_{\eta t_{i}}(x, y) \chi_{B_{i}}(y) d \mu(y) d \mu(x) \\
& \leq C \lambda<M(\varphi), \chi_{B_{i}}>
\end{aligned}
$$

where $\langle\cdot, \cdot\rangle$ denotes the inner product in complex space.

So,

$$
\left|\left\langle|\varphi|, \sum_{i}\left\|A_{t_{i}} b_{i}\right\|_{E}\right\rangle\right| \leq C \lambda<M(\varphi), \chi_{\bigcup_{i} B_{i}}>
$$

It follows that

$$
\begin{aligned}
& \left\|\sum_{i}\right\| A_{t_{i}} b_{i}\left\|_{E}\right\|_{L^{2}(\mathbb{X})} \leq C \lambda\left\|\sum_{i} \chi_{B_{i}}\right\|_{L^{2}(\mathbb{X})} \\
& \leq C \lambda\left(\sum_{i} \mu\left(B_{i}\right)\right)^{1 / 2} \leq C \lambda^{1 / 2}\|f\|_{L_{E}^{1}(\mathbb{X})}^{1 / 2}
\end{aligned}
$$

Therefore, by the Chebyshev inequality and the boundedness of $T$ on $L_{E}^{2}(\mathbb{X})$,

$$
\begin{aligned}
\mu\left(\left\{x \in \mathbb{X}:\left\|\sum_{i} T A_{t_{i}} b_{i}(x)\right\|_{F}>\lambda / 4\right\}\right) & \leq C \frac{1}{\lambda^{2}} \int_{\mathbb{X}}\left\|\sum_{i} T A_{t_{i}} b_{i}(x)\right\|_{F}^{2} d \mu(x) \\
& \leq C \frac{1}{\lambda^{2}} \int_{\mathbb{X}}\left\|\sum_{i} A_{t_{i}} b_{i}(x)\right\|_{E}^{2} d \mu(x) \\
& \leq C \frac{1}{\lambda^{2}}\left\|\sum_{i}\right\| A_{t_{i}} b_{i}\left\|_{E}\right\|_{L^{2}(\mathbb{X})}^{2} \\
& \leq C \frac{1}{\lambda}\|f\|_{L_{E}^{1}(\mathbb{X})} .
\end{aligned}
$$

On the other hand, set $\tilde{B}_{i}=\left(1+c_{1}\right) B_{i}$, where $c_{1}$ is the constant in (i) in Definition 2.1, then

$$
\begin{aligned}
& \mu\left(\left\{x \in \mathbb{X}:\left\|\sum_{i}\left(T-T A_{t_{i}}\right) b_{i}(x)\right\|_{F}>\lambda / 4\right\}\right) \\
& \leq \sum_{i} \mu\left(\tilde{B}_{i}\right)+\mu\left(\left\{x \in \mathbb{X} \backslash \cup_{i} \tilde{B}_{i}:\left\|\sum_{i}\left(T-T A_{t_{i}}\right) b_{i}(x)\right\|_{F}>\lambda / 4\right\}\right) \\
& \leq C \sum_{i} \mu\left(B_{i}\right)+\frac{4}{\lambda} \sum_{i} \int_{\mathbb{X} \backslash \cup_{i} \tilde{B}_{i}}\left\|\left(T-T A_{t_{i}}\right) b_{i}(x)\right\|_{F} d \mu(x) \\
& :=I+I I .
\end{aligned}
$$


Using the property (iii) of the Calderón-Zygmund decomposition, for $I$ we have

$$
I=C \sum_{i} \mu\left(B_{i}\right) \leq \frac{C}{\lambda}\|f\|_{L_{E}^{1}(\mathbb{X})}
$$

Since supp $b_{i} \subset B_{i}$ and $\tilde{B}_{i}=\left(1+c_{1}\right) B_{i}$, thus by assumption (i) in Definition 2.1 ,

$$
\begin{aligned}
I I & =\frac{4}{\lambda} \sum_{i} \int_{\mathbb{X} \backslash \cup_{i} \tilde{B}_{i}}\left\|\left(T-T A_{t_{i}}\right) b_{i}(x)\right\|_{F} d \mu(x) \\
& \leq \frac{4}{\lambda} \sum_{i} \int_{\mathbb{X} \backslash \cup_{i} \tilde{B}_{i}}\left\|\int_{\mathbb{X}}\left(K(x, y)-k_{t_{i}}(x, y)\right) b_{i}(y) d \mu(y)\right\|_{F} d \mu(x) \\
& \leq \frac{4}{\lambda} \sum_{i} \int_{\mathbb{X}}\left\|b_{i}(y)\right\|_{E}\left(\int_{d(x, y) \geq c_{1} t_{i}^{1 / m}}\left\|K(x, y)-k_{t_{i}}(x, y)\right\|_{\mathcal{L}(E, F)} d \mu(x)\right) d \mu(y) \\
& \leq \frac{C}{\lambda} \sum_{i}\left\|b_{i}\right\|_{L_{E}^{1}(\mathbb{X})} \leq \frac{C}{\lambda}\|f\|_{L_{E}^{1}(\mathbb{X})},
\end{aligned}
$$

Combining (2.2), (2.4), (2.5), (2.6) with (2.7), we show that $T$ is an operator of weak type $(1,1)$. By the Marcinkiewicz interpolation theorem, $T$ is bounded from $L_{E}^{p}(\mathbb{X})$ to $L_{F}^{p}(\mathbb{X})$ for $1<p \leq 2$.

But for $2<p<\infty$, we must pass to the adjoint operator. However, for $1 \leq$ $p<\infty, L_{E^{*}}^{p^{\prime}}(\mathbb{X}) \neq\left(L_{E}^{p}(\mathbb{X})\right)^{*}$, in general. In fact, if $f \in L_{E}^{p}(\mathbb{X})$ and $g \in L_{E^{*}}^{p^{\prime}}(\mathbb{X})$, then the active $\langle f, g\rangle(x)=<f(x), g(x)>$ is integrable; furthermore,

$$
\|g\|_{L_{E^{*}}^{p^{\prime}}(\mathbb{X})}=\sup \left\{\left|\int_{\mathbb{X}}<f(x), g(x)>d \mu(x)\right|:\|f\|_{L_{E}^{p}(\mathbb{X})} \leq 1\right\} .
$$

From this we see that $L_{E^{*}}^{p^{\prime}}(\mathbb{X}) \subset\left(L_{E}^{p}(\mathbb{X})\right)^{*}$.

In the following we will show how to pass to the adjoint operator, which comes from [13].

When $E$ is reflexive $L_{E^{*}}^{p^{\prime}}(\mathbb{X})=\left(L_{E}^{p}(\mathbb{X})\right)^{*}$, it is enough to note that the kernel associated with the adjoin operator $T^{*}$ is $K(y, x)=K^{*}(x, y) \in \mathcal{L}\left(F^{*}, E^{*}\right)$. In Definition 2.1 the condition (ii) for $K$ is equivalent to (i) for $K^{*}$, so repeating the above argument we get that $T^{*}$ is bounded for $1<p \leq 2$.

When $L_{E^{*}}^{p^{\prime}}(\mathbb{X}) \neq\left(L_{E}^{p}(\mathbb{X})\right)^{*}$, we must consider the finite dimensional subspaces of $E$.

If $f$ is a scalar function in $L^{p}(\mathbb{X})$ and $b \in E$, define the function $f \cdot b$ from $\mathbb{X}$ to $E$ by $(f \cdot b)(x)=f(x) b$. This function is in $L_{E}^{p}(\mathbb{X})$ and its norm is $\|f\|_{L^{p}(\mathbb{X})}\|b\|_{E}$. The subspace of $L_{E}^{p}(\mathbb{X})$ consisting of finite linear combinations of function of this type, denote by $L^{p} \otimes E$, is dense if $1 \leq p<\infty$. Given such a subspace $E_{0}$, let 
$T_{0}: L_{E_{0}}^{2}(\mathbb{X}) \longmapsto L_{F}^{2}(\mathbb{X})$ be the restriction of $T$ to functions with values in $E_{0}$. The kernel associated with $T_{0}$ is $K_{0} \in \mathcal{L}\left(E_{0}, F\right)$, which is the restriction of $K$ on $E_{0}$. Since $\left\|K_{0}\right\|_{\mathcal{L}\left(E_{0}, F\right)} \leq\|K\|_{\mathcal{L}(E, F)}$, inequalities (i) and (ii) in Definition 2.1 hold for $K_{0}$ with constants independent of the subspaces $E_{0}$. Therefore, arguing as before,

$$
T_{0}^{*}: L_{F^{*}}^{q} \longmapsto L_{E_{0}^{*}}^{q}, \text { for } 1<q \leq 2 .
$$

So by duality, $T_{0}$ is bounded from $L_{E_{0}}^{p}(\mathbb{X})$ to $L_{F}^{p}(\mathbb{X})$ for $2 \leq p<\infty$. Since $L_{E}^{p} \otimes E$ is dense in $L_{E}^{p}(\mathbb{X})$, we get the desired result.

For the proof of (iii), it is sufficient for us to show that for any $f \in L_{E}^{2}(\mathbb{X}) \cap$ $L_{E}^{\infty}(\mathbb{X})$ and $x \in \mathbb{X}$

$$
\sup _{B \ni x} \frac{1}{\mu(B)} \int_{B}\left\|T f(y)-A_{t_{B}}^{\prime} T f(y)\right\|_{F} d \mu(y) \leq C\|f\|_{L_{E}^{\infty}(\mathbb{X})},
$$

in which $t_{B}=r_{B}^{m}$ and $r_{B}$ is the radius of $B$.

Let $f \in L_{E}^{2}(\mathbb{X}) \cap L_{E}^{\infty}(\mathbb{X})$ be given and $B=B\left(x_{0}, r_{B}\right) \ni x$. We write $f_{1}=f \chi_{2 B}$ and $f_{2}=f-f_{1}$, then

$$
T f-A_{t_{B}}^{\prime} T f=\left(T f_{1}-A_{t_{B}}^{\prime} T f_{1}\right)+\left(T-A_{t_{B}}^{\prime} T\right) f_{2} .
$$

Thus,

$$
\begin{aligned}
& \int_{B}\left\|T f(y)-A_{t_{B}}^{\prime} T f(y)\right\|_{F} d \mu(y) \\
& \quad \leq \int_{B}\left\|T f_{1}(y)-A_{t_{B}}^{\prime} T f_{1}(y)\right\|_{F} d \mu(y)+\int_{B}\left\|\left(T-A_{t_{B}}^{\prime} T\right) f_{2}(y)\right\|_{F} d \mu(y) \\
& \quad:=I+I I
\end{aligned}
$$

Let us estimate $I$ and $I I$ respectively.

By (1.4) and Lemma 2.6,

$$
\begin{aligned}
& \left\|A_{t_{B}}^{\prime} T f_{1}(y)\right\|_{F} \\
& =\left\|\int_{\mathbb{X}} a_{t_{B}}^{\prime}(y, z) T_{1} f(z) d \mu(z)\right\|_{F} \\
& \leq \int_{\mathbb{X}}\left\|a_{t_{B}}^{\prime}(y, z)\right\|_{\mathcal{L}(F)}\left\|T f_{1}(z)\right\|_{F} d \mu(z) \\
& \leq \int_{\mathbb{X}} h_{t_{B}}(y, z)\left\|T f_{1}(z)\right\|_{F} d \mu(z) \\
& \leq C M\left(\left\|T f_{1}\right\|_{F}\right)(y)
\end{aligned}
$$


So,

$$
\begin{aligned}
I & \leq C \int_{B} M\left(\left\|T f_{1}\right\|_{F}\right)(y) d \mu(y) \\
& \leq C(\mu(B))^{1 / 2}\left(\int_{B}\left(M\left(\left\|T f_{1}\right\|_{F}\right)(y)\right)^{2} d \mu(y)\right)^{1 / 2} \\
& \leq C(\mu(B))^{1 / 2}\left(\int_{B}\left\|T f_{1}(y)\right\|_{F}^{2} d \mu(y)\right)^{1 / 2} \\
& \leq C(\mu(B))^{1 / 2}\left(\int_{2 B}\|f(y)\|_{E}^{2} d \mu(y)\right)^{1 / 2} \\
& \leq C \mu(B)\|f\|_{L_{E}^{\infty}(\mathbb{X})} .
\end{aligned}
$$

By the assumption (ii) in Definition 2.1,

$$
\begin{aligned}
I I & \leq \int_{B} \int_{\mathbb{X} \backslash 2 B}\left\|K(y, z)-K_{t}(y, z)\right\|_{\mathcal{L}(E, F)}\|f(z)\|_{E} d \mu(z) d \mu(y) \\
& \leq C\|f\|_{L_{E}^{\infty}(\mathbb{X})} \int_{B} \int_{\mathbb{X} \backslash 2 B}\left\|K(y, z)-K_{t}(y, z)\right\|_{\mathcal{L}(E, F)} d \mu(z) d \mu(y) \\
& \leq C \mu(B)\|f\|_{L_{E}^{\infty}(\mathbb{X})} .
\end{aligned}
$$

Thus, for $f \in L_{E}^{2}(\mathbb{X}) \cap L_{E}^{\infty}(\mathbb{X})$, we get the desired result.

\section{MAXimal tRUNCATED OPERATOR}

Let $T$ be a linear operator mapping $E$-valued functions into $F$-valued functions. If $T$ is bounded from $L_{E}^{2}(\mathbb{X})$ to $L_{F}^{2}(\mathbb{X})$ and the associated kernel $K(x, y)$ : $\mathbb{X} \times \mathbb{X} \longmapsto \mathcal{L}(E, F)$ satisfies the following conditions:

(i) There exists an "approximation to identity" $\left\{A_{t}: t>0\right\} \subset \mathcal{L}(E)$ such that $T A_{t}$ has an associated kernel $k_{t}(x, y)$, and there exist constants $c_{2}, C>0$, so that

$$
\int_{d(x, y) \geq c_{2} t^{1 / m}}\left\|K(x, y)-k_{t}(x, y)\right\|_{\mathcal{L}(E, F)} d \mu(x) \leq C, \quad \text { for all } y \in \mathbb{X} .
$$

(ii) There exists an "approximation to identity" $\left\{A_{t}^{\prime}: t>0\right\} \subset \mathcal{L}(F)$ such that $A_{t}^{\prime} T$ has an associated kernel $K_{t}(x, y)$, and there is a constant $c_{4}>0$, so that

(a) $\left\|K_{t}(x, y)\right\|_{\mathcal{L}(E, F)} \leq c_{4} \frac{1}{\mu\left(B\left(x, t^{1 / m}\right)\right)}$

and

(b) $\left\|K(x, y)-K_{t}(x, y)\right\|_{\mathcal{L}(E, F)} \leq c_{4} \frac{1}{\mu(B(x, d(x, y)))} \omega\left(\frac{t^{1 / m}}{d(x, y)}\right)$,

when $d(x, y) \geq c_{3} t^{1 / m}$, where $\omega$ is an increasing function satisfying the Dini-type condition $\int_{0}^{1} \omega(t) \frac{d t}{t}<\infty$. 
Remark 4. In the scalar case, take $\omega=r^{\alpha}$ then (b) is equivalent to (19) in [4], so the condition assumed on $K$ is generalized in the paper. In addition, $\int_{0}^{1} \omega(t) \frac{d t}{t}<\infty$ is equivalent to the discrete form $\sum_{k=0}^{\infty} \omega\left(2^{-k}\right)<\infty$.

Then define maximal truncated operator

$$
T_{*} f(x)=\sup _{\epsilon>0}\left\|T_{\epsilon} f(x)\right\|_{F},
$$

where $T_{\epsilon}$ is the truncated singular operator defined by

$$
T_{\epsilon}=\int_{d(x, y) \geq \epsilon} K(x, y) f(y) d \mu(y), \quad \text { for some } \epsilon>0 .
$$

Theorem 3.1. Assume that $T_{*}$ is as above, then there exists a constant $C>0$ such that

$$
T_{*} f(x) \leq C\left(M\left(\|T f\|_{F}\right)(x)+M\left(\|f\|_{E}\right)(x)\right) .
$$

Theorem 3.2. Assume that $T_{*}$ is as above, then $T_{*}$ is bounded from $L_{E}^{p}(\mathbb{X})$ to $L^{p}(\mathbb{X})$ for all $1<p<\infty$.

It is not hard to check that in this section $T$ satisfies the condition (i) and (ii) of Definition 2.1, so $T$ is bounded from $L_{E}^{p}(\mathbb{X})$ to $L_{F}^{p}(\mathbb{X})$ for $1<p<\infty$. Thus by Theorem 3.1, Theorem 3.2. can be proved. So we need only to prove Theorem 3.1. Without loss of generality, we assume that $c_{3}=1$.

For a fixed $\epsilon>0$, write

$$
T_{\epsilon} f(x)=A_{\epsilon^{m}}^{\prime} T f(x)-\left(A_{\epsilon^{m}}^{\prime} T-T_{\epsilon}\right) f(x) .
$$

Since

$$
\begin{aligned}
\left\|A_{\epsilon^{m}}^{\prime} T f(x)\right\|_{F} \leq & \int_{\mathbb{X}}\left\|a_{\epsilon^{m}}^{\prime}(x, y)\right\|_{\mathcal{L}(F)}\|T f(y)\|_{F} d \mu(y) \\
& \leq \int_{\mathbb{X}} h_{\epsilon^{m}}(x, y)\|T f(y)\|_{F} d \mu(y) .
\end{aligned}
$$

Thus by Lemma 2.6, we have

$$
\left\|A_{\epsilon^{m}}^{\prime} T f(x)\right\|_{E} \leq C M\left(\|T f\|_{F}\right)(x) .
$$

On the other hand, the kernel of the operator $A_{\epsilon}^{\prime} T-T_{\epsilon}$ is given by $K_{\epsilon} m(x, y)-$ $\bar{K}_{\epsilon}(x, y)$, in which $\bar{K}_{\epsilon}(x, y)=K(x, y)$ if $d(x, y) \geq \epsilon$ and $\bar{K}_{\epsilon}(x, y)=0$ otherwise. So, there are two cases:

case (i) $d(x, y)<\epsilon$, then $K_{\epsilon}(x, y)=0$ and it follows from (a) that

$$
\left\|K_{\epsilon^{m}}(x, y)-\bar{K}_{\epsilon}(x, y)\right\|_{\mathcal{L}(E, F)}=\left\|K_{\epsilon^{m}}(x, y)\right\|_{\mathcal{L}(E, F)} \leq c_{4} \frac{1}{\mu(B(x, \epsilon))},
$$


case (ii) $d(x, y) \geq \epsilon$, then $\bar{K}_{\epsilon}(x, y)=K(x, y)$ and it follows from (b) that

$$
\left\|K_{\epsilon^{m}}(x, y)-\bar{K}_{\epsilon}(x, y)\right\|_{\mathcal{L}(E, F)} \leq c_{4} \frac{1}{\mu(B(x, d(x, y)))} \omega\left(\frac{\epsilon}{d(x, y)}\right) .
$$

Therefore, using $\sum_{k=0}^{\infty} \omega\left(2^{-k}\right)<\infty$,

$$
\begin{aligned}
\| & \left(A_{\epsilon^{m}}^{\prime} T-T_{\epsilon}\right) f(x) \|_{F} \\
= & \left\|\int_{\mathbb{X}}\left(K_{\epsilon^{m}}(x, y)-\bar{K}_{\epsilon}(x, y)\right) f(y) d \mu(y)\right\|_{F} \\
\leq & \int_{\mathbb{X}}\left\|K_{\epsilon^{m}}(x, y)-\bar{K}_{\epsilon}(x, y)\right\|_{\mathcal{L}(E, F)}\|f(y)\|_{E} d \mu(y) \\
\leq & C \frac{1}{\mu(B(x, \epsilon))} \int_{d(x, y)<\epsilon}\|f(y)\|_{E} d \mu(y) \\
& +C \int_{d(x, y) \geq \epsilon} \frac{1}{\mu(B(x, d(x, y)))} \omega\left(\frac{\epsilon}{d(x, y)}\right)\|f(y)\|_{E} d \mu(y) \\
\leq & C \frac{1}{\mu(B(x, \epsilon))} \int_{d(x, y)<\epsilon}\|f(y)\|_{E} d \mu(y) \\
& +C \sum_{k=0}^{\infty} \int_{2^{k} \epsilon \leq d(x, y)<2^{k+1} \epsilon} \overline{\mu(B(x, d(x, y)))} \omega\left(\frac{\epsilon}{d(x, y)}\right)\|f(y)\|_{E} d \mu(y) \\
\leq & C \frac{1}{\mu(B(x, \epsilon))} \int_{d(x, y) \leq \epsilon}\|f(y)\|_{E} d \mu(y) \\
& +C \sum_{k=0}^{\infty} \omega\left(2^{-k}\right) \frac{1}{\mu\left(B\left(x, 2^{k+1} \epsilon\right)\right)} \int_{d(x, y) \leq 2^{k+1} \epsilon}\|f(y)\|_{E} d \mu(y) \\
\leq & C M\left(\|f\|_{E}\right)(x) .
\end{aligned}
$$

Combining the estimates of (3.3) with (3.4), we have

$$
T_{*} f(x)=\sup _{\epsilon>0}\left\|T_{\epsilon} f(x)\right\|_{F} \leq C\left(M\left(\|T f\|_{F}\right)(x)+M\left(\|f\|_{E}\right)(x)\right) .
$$

Thus we finish the proof of Theorem 3.1.

\section{Multilinear commutators}

4.1. Some notations and the main results. Let $\mathbb{X}$ be a space of homogeneous type with a distance $d$ and a non-negative Borel regular measure $\mu$. If for any $x \in \mathbb{X}$ and $r>0$, where $\mu(x)<r^{n}<\mu(\mathbb{X})$, there exist $0<c_{1} \leq c_{2}<\infty$, such that $c_{1} r^{n} \leq \mu(B(x, r)) \leq c_{2} r^{n}$, then $(\mathbb{X}, d, \mu)$ is said to be normal. It is obvious that the condition of above means the doubling condition (1.2). 
Definition 4.1.1 A locally integrable function $b$ on $\mathbb{X}$ is said to be in $B M O$ if and only if

$$
\sup _{x \in B} \frac{1}{\mu(B)} \int_{B}\left|b(y)-b_{B}\right| d \mu(y)<\infty
$$

where $b_{B}=\frac{1}{\mu(B)} \int_{B} b(y) d \mu(y)$. The $B M O$ norm of $b$ is defined by

$$
\|b\|_{*}=\sup _{B} \frac{1}{\mu(B)} \int_{B}\left|b(y)-b_{B}\right| d \mu(y)<\infty .
$$

Definition 4.1.2.([2]) Let $0<\beta<1$, the Lipschitz space on space of homogenous type $\mathbb{X}$ is the space of functions $f$ such that

$$
\|f\|_{L i p(\beta)}=\sup _{x, y \in \mathbb{X}, x \neq y} \frac{|f(x)-f(y)|}{d(x, y)^{\beta}}<\infty .
$$

Definition 4.1.3. Let $T$, mapping $E$-valued functions into $F$-valued functions, be a singular integral operator with non-smooth kernel $K(x, y): \mathbb{X} \times \mathbb{X} \longmapsto(E, F)$ satisfying:

(i) $T$ is bounded from $L_{E}^{2}(\mathbb{X})$ to $L_{F}^{2}(\mathbb{X})$ and for any $f \in L_{c, E}^{\infty}(\mathbb{X})$,

$$
T f(x)=\int_{\mathbb{X}} K(x, y) f(y) d \mu(y) \text { for a.e } x \notin \operatorname{supp} f .
$$

(ii) There exists an "approximation to identity" $\left\{A_{t}: t>0\right\} \subset \mathcal{L}(E)$ such that $T A_{t}$ has an associated kernel $k_{t}(x, y)$. And there exist constants $c_{5}, C>0$, so that

$$
\int_{d(x, y) \geq c_{5} t^{1 / m}}\left\|K(x, y)-k_{t}(x, y)\right\|_{\mathcal{L}(E, F)} d \mu(x) \leq C, \quad \text { for all } y \in \mathbb{X}
$$

(iii) There exist constants $C>0,0<\delta<1$ and an "approximation to identity" $\left\{A_{t}^{\prime}: t>0\right\} \subset \mathcal{L}(F)$ such that $A_{t}^{\prime} T$ has an associated kernel $K_{t}(x, y)$ satisfying

$$
\left\|K(x, y)-K_{t}(x, y)\right\|_{\mathcal{L}(E, F)} \leq C \frac{1}{\mu(B(x, d(x, y)))} \frac{t^{\delta / m}}{d(x, y)^{\delta}},
$$

for $d(x, y) \geq c_{5}^{\prime} t^{1 / m}$. In what follows we will assume that $c_{5}=c_{5}^{\prime}=1$.

Let $l \in \mathbb{N}$ is finite. Suppose that $\vec{b}=\left(b_{1}, b_{2}, \ldots, b_{l}\right)$ is a finite family of locally integrable functions, then the multilinear commutator generated by $T$ and $\vec{b}$ is defined by

$$
T_{\vec{b}} f(x)=\left[b_{l}, \ldots,\left[b_{2},\left[b_{1}, T\right]\right], \ldots,\right] f(x) .
$$

It is obvious that when $l=1, T_{\vec{b}} f(x)=\left[b_{1}, T\right] f(x)=b_{1}(x) T f(x)-T\left(b_{1} f\right)(x)$ is the commutator generated by $T$ and $b_{1}$, and when $b_{1}=\cdots=b_{l}, T_{\vec{b}} f$ is the higher commutator. 
Remark 5. From Theorem 2.3, it is easy to see that the operator $T$ in Definition 4.1.3 is bounded from $L_{E}^{p}(\mathbb{X})$ to $L_{F}^{p}(\mathbb{X})$ for all $1<p<\infty$.

Remark 6. Given any positive integer $l$, for all $1 \leq j \leq l$, we denote the family of all finite subsets $\sigma=\left\{\sigma_{1}, \sigma_{2}, \cdots, \sigma_{j}\right\}$ of $\{1,2, \ldots, l\}$ of different elements by $C_{j}^{l}$ and for any $\sigma \in C_{j}^{l}$, let $\sigma^{\prime}=\{1,2, \cdots, l\} \backslash \sigma$. Let $\vec{b}=\left(b_{1}, b_{2}, \cdots, b_{l}\right)$ be a finite function family then for any $\sigma=\left\{\sigma_{1}, \sigma_{2}, \cdots, \sigma_{j}\right\} \in C_{j}^{l}$, we denote $\vec{b}_{\sigma}=\left\{b_{\sigma_{1}}, b_{\sigma_{2}}, \cdots, b_{\sigma_{j}}\right\}$ and $b_{\sigma}(x)=\prod_{i=1}^{j} b_{\sigma_{i}}(x)$. With these notations, for any $l$-tuple $\left(\beta_{1}, \cdots, \beta_{l}\right)$ of positive numbers, we set $\beta_{\sigma}=\sum_{i=1}^{j} \beta_{\sigma_{i}},\left\|\vec{b}_{\sigma}\right\|_{*}=\prod_{i=1}^{j}\left\|b_{\sigma_{i}}\right\|_{*}$, and $\left\|\vec{b}_{\sigma}\right\|_{L i p\left(\beta_{\sigma}\right)}=\prod_{i=1}^{j}\left\|b_{\sigma_{i}}\right\|_{L i p\left(\beta_{\sigma_{i}}\right)}$. Denote $\beta=\sum_{j=1}^{l} \beta_{j},\|\vec{b}\|_{*}=\prod_{j=1}^{l}\left\|b_{j}\right\|_{*}$ and $\|\vec{b}\|_{L i p(\beta)}=\prod_{j=1}^{l}\left\|b_{j}\right\|_{L i p\left(\beta_{j}\right)}$, simply.

Theorem 4.1.4. Let $1<p<\infty$ and $T_{\vec{b}}$ be as above. If $b_{j} \in B M O$ for all $1 \leq j \leq l$, then $T_{\vec{b}}$ is bounded from $L_{E}^{p}(\mathbb{X})$ to $L_{F}^{p}(\mathbb{X})$.

Theorem 4.1.5. Suppose that $(\mathbb{X}, d, \mu)$ is normal. Let $T_{\vec{b}}$ be as above. If $0<$ $\beta_{1}, \beta_{2}, \ldots, \beta_{l}<1$ such that $0<\sum_{j=1}^{l} \beta_{j}=\beta<n$, and $b_{j} \in \operatorname{Lip}\left(\beta_{j}\right)(1 \leq j \leq l)$, then $T_{\vec{b}}$ is bounded from $L_{E}^{p}(\mathbb{X})$ to $L_{F}^{q}(\mathbb{X})$, here $1<p<n / \beta$ such that $1 / q=$ $1 / p-\beta / n$.

When $l=1$, denote $b_{1}=b$ and $\beta_{1}=\beta$, then we have the following result.

Theorem 4.1.6 Suppose that $(\mathbb{X}, d, \mu)$ is normal. Let $0<\delta<1$ be as in Definition 4.1.3. If $0<\beta<1$ and $n / \beta \leq p<\infty$ such that $\delta / n-(\beta / n-1 / p)>0$, then for $b \in \operatorname{Lip}(\beta)$ there exists a constant $C>0$ such that

$$
\sup _{B} \frac{1}{\mu(B)^{1+\beta / n-1 / p}} \int_{B}\left\|T_{b} f(x)-A_{t_{B}}^{\prime}\left(T_{b} f\right)(x)\right\|_{F} d \mu(x) \leq C\|b\|_{L i p(\beta)}\|f\|_{L_{E}^{p}(\mathbb{X})} .
$$

Remark 7 Let $\mathbb{X}=\mathbb{R}^{n}$ is Euclidean space. In the scalar field, if $p=n / \beta$, Theorem 4.1.6 implies that $T_{b}$ is bounded from $L^{p}\left(\mathbb{R}^{n}\right)$ to $B M O_{A^{\prime}}$.

4.2. The proof of Theorem 4.1.4. To prove Theorem 4.1.4 we need the following Lemmas.

Lemma 4.2.1([14]) Assume that $b \in B M O$ and $k>1$. Then for every ball $B$, we have

$$
\left|b_{B}-b_{2^{k} B}\right| \leq C k\|b\|_{*},
$$

where $2^{k} B$ denotes the $2^{k}$ times extensions of $B$ with the same center. 
Lemma 4.2.2. Let $b_{j} \in B M O(j=1,2, \ldots, l)$, then for any $\sigma \in C_{j}^{l}(j=$ $1,2, \ldots, l)$ and $1 \leq \gamma<\infty$,

$$
\left(\frac{1}{\mu(B)} \int_{B}\left|\left(b(y)-b_{B}\right)_{\sigma}\right|^{\gamma} d \mu(y)\right)^{1 / \gamma} \leq C\left\|\vec{b}_{\sigma}\right\|_{*} .
$$

Proof Take $\gamma \leq \gamma_{1}, \gamma_{2}, \ldots, \gamma_{j}<\infty$ such that $1 / \gamma_{1}+1 / \gamma_{2}+\cdots+1 / \gamma_{j}=1 / \gamma$, then by Hölder's inequality

$$
\begin{aligned}
& \left(\frac{1}{\mu(B)} \int_{B}\left|\left(b(y)-b_{B}\right)_{\sigma}\right|^{\gamma} d \mu(y)\right)^{1 / \gamma} \\
& \leq C \prod_{i=1}^{j}\left(\frac{1}{\mu(B)} \int_{B}\left|b_{\sigma_{i}}(y)-\left(b_{\sigma_{i}}\right)_{B}\right|^{\gamma_{i}} d \mu(y)\right)^{1 / \gamma_{i}} \\
& \leq C\left\|\vec{b}_{\sigma}\right\|_{*} .
\end{aligned}
$$

Lemma 4.2.3. Let $H$ be a Banach space. Assume that $\left\{A_{t}: t>0\right\}$ is an "approximation to identity" on $H$ and $b_{j} \in B M O(j=1,2, \ldots, l)$. Then for every $f \in L_{H}^{p}(1<p<\infty), \sigma \in C_{j}^{l}(j=1,2, \ldots, l)$ and $1<\gamma<\infty$, there is a constant $C>0$ such that

$$
\sup _{B \ni x} \frac{1}{\mu(B)} \int_{B}\left\|A_{t_{B}}\left(\left(b-b_{B}\right)_{\sigma} f\right)(y)\right\|_{H} d \mu(y) \leq C\left\|\vec{b}_{\sigma}\right\|_{*} M_{\gamma}\left(\|f\|_{H}\right)(x),
$$

where $t_{B}=r_{B}^{m}$ and $r_{B}$ is the radius of $\mathrm{B}$.

Proof For $1<p<\infty$, fixed $f \in L_{H}^{p}(\mathbb{X})$. Let $B=B\left(x_{0}, r_{B}\right) \ni x$, then

$$
\begin{aligned}
& \frac{1}{\mu(B)} \int_{B}\left\|A_{t_{B}}\left(\left(b-b_{B}\right)_{\sigma} f\right)(y)\right\|_{H} d \mu(y) \\
\leq & \frac{1}{\mu(B)} \int_{B} \int_{\mathbb{X}}\left\|a_{t_{B}}(y, z)\right\|_{\mathcal{L}(H)}\left\|\left(b(z)-b_{B}\right)_{\sigma} f(z)\right\|_{H} d \mu(z) d \mu(y) \\
\leq & \frac{1}{\mu(B)} \int_{B} \int_{{ }_{B}} h_{t_{B}}(y, z)\left\|\left(b(z)-b_{B}\right)_{\sigma} f(z)\right\|_{H} d \mu(z) d \mu(y) \\
& +\frac{1}{\mu(B)} \int_{B} \sum_{k=1}^{\infty} \int_{2^{k+1} B \backslash 2^{k} B} h_{t_{B}}(y, z)\left\|\left(b(z)-b_{B}\right)_{\sigma} f(z)\right\|_{H} d \mu(z) d \mu(y) \\
:= & I+I I .
\end{aligned}
$$

Notice that for any $y \in B$ and $z \in 2 B$, by (1.5) and the properties of function $s$,

$$
h_{t_{B}}(y, z) \leq C s(0) \mu(2 B)^{-1} .
$$

Thus

$$
I \leq C \frac{1}{\mu(2 B)} \int_{2 B}\left|\left(b(z)-b_{B}\right)_{\sigma}\right|\|f(z)\|_{H} d \mu(z) .
$$


On the other hand, take $1<\gamma, \gamma^{\prime}<\infty$ which satisfies $1 / \gamma+1 / \gamma^{\prime}=1$, then by Hölder's inequality, Lemma 4.2.1 and Lemma 4.2.2,

$$
\begin{aligned}
I & \leq C\left(\frac{1}{\mu(2 B)} \int_{2 B}\left|\left(b(z)-b_{B}\right)_{\sigma}\right|^{\gamma^{\prime}} d \mu(z)\right)^{1 / \gamma^{\prime}}\left(\frac{1}{\mu(2 B)} \int_{2 B}\|f(z)\|_{E}^{\gamma} d \mu(z)\right)^{\gamma} \\
& \leq C\left\|\vec{b}_{\sigma}\right\|_{*} M_{\gamma}\left(\|f\|_{E}\right)(x) .
\end{aligned}
$$

Moreover, for any $y \in B$ and $z \in 2^{k+1} B \backslash 2^{k} B$, we have $d(y, z) \geq 2^{k-1} r_{B}$. So, using (1.5) and (1.3),

$$
h_{t_{B}}(y, z)=\frac{s\left(d(y, z)^{m} t^{-1}\right)}{\mu\left(B\left(y, t^{1 / m}\right)\right)} \leq C \frac{s\left(2^{(k-1) m}\right) 2^{(k+1) n}}{\mu\left(2^{k+1} B\right)} .
$$

Thus by Lemma 4.2.2 and (4.2.3), it is similar to the estimate of (4.2.2),

$$
\begin{aligned}
I I \leq & C \sum_{k=1}^{\infty} \frac{s\left(2^{(k-1) m}\right) 2^{(k+1) n}}{\mu\left(2^{k+1} B\right)} \int_{2^{k+1} B}\left|\left(b(z)-b_{B}\right)_{\sigma}\right|\|f(z)\|_{H} d \mu(z) \\
\leq & C \sum_{k=1}^{\infty} 2^{(k+1) n} s\left(2^{(k-1) m}\right)\left(\frac{1}{\mu\left(2^{k+1} B\right)} \int_{2^{k+1} B}\left|\left(b(z)-b_{B}\right)_{\sigma}\right|^{\gamma^{\prime}} d \mu(z)\right)^{1 / \gamma^{\prime}} \\
& \times\left(\frac{1}{\mu\left(2^{k+1} B\right)} \int_{2^{k+1} B}\|f(z)\|_{H}^{\gamma} d \mu(z)\right)^{1 / \gamma} \\
\leq & C\left\|\vec{b}_{\sigma}\right\|_{*} M_{\gamma}\left(\|f\|_{H}\right)(x),
\end{aligned}
$$

where $\gamma$ is the same as in (4.2.2). Thus we complete the proof of Lemma 4.2.3.

Lemma 4.2.4. Let $H$ be a Banach space. Assume that $\mu(\mathbb{X})=\infty$ and $f \in L_{H}^{p}(\mathbb{X})$ for $1<p<\infty$. Then for every $0<\eta<1$ and any $0<\lambda$, there exists a constant $C_{\mathbb{X}}>0$ and $\gamma>0$ (independent of $\lambda$ and $f$ ), such that

$$
\begin{aligned}
& \mu\left(\left\{x \in \mathbb{X}: M\left(\|f\|_{H}\right)(x)>D \lambda, M_{A}^{\#}\left(\|f\|_{H}\right)(x) \leq \gamma \lambda\right\}\right) \\
& \leq C \eta \mu\left(\left\{x \in \mathbb{X}: M\left(\|f\|_{H}\right)(x)>\lambda\right\}\right),
\end{aligned}
$$

where $D$ is a fixed constant which only depends on the space $\mathbb{X}$ and the "approximation to identity" $\left\{A_{t}, t>0\right\}$. As a consequence, we have

$$
\|f\|_{L_{H}^{p}(\mathbb{X})} \leq\left\|M\left(\|f\|_{H}\right)\right\|_{L^{p}(\mathbb{X})} \leq C\left\|M_{A}^{\#}\left(\|f\|_{H}\right)\right\|_{L^{p}(\mathbb{X})} .
$$

In the scalar case, it is Martell who give the proof of Lemma 4.2.4(see [10]). In fact by some modifications for the proofs of Proposition 4.1 and Theorem 4.2 in [10], one can prove Lemma 4.2.4. We omit the proof here for brevity.

Let us now turn to prove Theorem 4.1.4. 
To prove Theorem 4.1.4, we claim that for any $x \in \mathbb{X}$,

$$
\begin{aligned}
M_{A^{\prime}}^{\#}\left(\left\|T_{\vec{b}} f\right\|_{F}\right)(x) \leq & C\left(\|\vec{b}\|_{*} M_{\tau}\left(\|T f\|_{F}\right)(x)+\|\vec{b}\|_{*} M_{\gamma \xi}\left(\|f\|_{E}\right)(x)\right. \\
& \left.+\sum_{j=1}^{l-1} \sum_{\sigma \in C_{j}^{l}} C_{j, l}\left\|\vec{b}_{\sigma}\right\|_{*} M_{\tau}\left(\left\|T_{\vec{b}_{\sigma^{\prime}}} f\right\|_{F}\right)(x)\right)
\end{aligned}
$$

where $1<\tau<p$ and $1<\gamma, \xi<\infty$, such that $1<\gamma \xi<p$.

In fact, for any $x \in \mathbb{X}$, take $B=B\left(x_{0}, r_{B}\right) \ni x$. Fix $f \in L_{E}^{p}(\mathbb{X})$, we set $f_{1}=$ $f \chi_{2 B}$ and $f_{2}=f-f_{1}$. And denote $\vec{\lambda}=\left(\lambda_{1}, \lambda_{2}, \ldots, \lambda_{l}\right)=\left(\left(b_{1}\right)_{B},\left(b_{2}\right)_{B}, \ldots,\left(b_{l}\right)_{B}\right)=$ $\vec{b}_{B}$. Then $T_{\vec{b}} f$ can be written as the following form

$$
\begin{aligned}
T_{\vec{b}} f(y)= & \int_{\mathbb{X}} \prod_{j=1}^{l}\left(b_{j}(y)-b_{j}(z)\right) K(y, z) f(z) d \mu(z) \\
= & \int_{\mathbb{X}} \prod_{j=1}^{l}\left(\left(b_{j}(y)-\lambda_{j}\right)-\left(b_{j}(z)-\lambda_{j}\right)\right) K(y, z) f(z) d \mu(z) \\
= & \sum_{j=0}^{l} \sum_{\sigma \in C_{j}^{l}}(-1)^{l-j}(b(y)-\lambda)_{\sigma} \int_{\mathbb{X}}(b(z)-\lambda)_{\sigma^{\prime}} K(y, z) f(z) d \mu(z) \\
= & \prod_{j=1}^{l}\left(b_{j}(y)-\lambda_{j}\right) T f(y) \\
& +\sum_{j=1}^{l-1} \sum_{\sigma \in C_{j}^{l}}(-1)^{l-j}(b(y)-\lambda)_{\sigma} \int_{\mathbb{X}}(b(z)-\lambda)_{\sigma^{\prime}} K(y, z) f(z) d \mu(z) \\
& +(-1)^{l} T\left(\prod_{j=1}^{l}\left(b_{j}-\lambda_{j}\right) f\right)(y) .
\end{aligned}
$$

Now expanding $(b(z)-\lambda)_{\sigma^{\prime}}=((b(z)-b(y))+(b(y)-\lambda))_{\sigma^{\prime}}$, it is easy to see that

$$
\begin{aligned}
T_{\vec{b}} f(y)= & \prod_{j=1}^{l}\left(b_{j}(y)-\lambda_{j}\right) T f(y)+\sum_{j=1}^{l-1} \sum_{\sigma \in C_{j}^{l}} C_{j, l}(b(y)-\lambda)_{\sigma} T_{\vec{b}_{\sigma^{\prime}}} f(y) \\
& +(-1)^{l} T\left(\prod_{j=1}^{l}\left(b_{j}-\lambda_{j}\right) f_{1}\right)(y)+(-1)^{l} T\left(\prod_{j=1}^{l}\left(b_{j}-\lambda_{j}\right) f_{2}\right)(y),
\end{aligned}
$$


and

$$
\begin{aligned}
A_{t_{B}}^{\prime}\left(T_{\vec{b}} f\right)(y)= & A_{t_{B}}^{\prime}\left(\prod_{j=1}^{l}\left(b_{j}-\lambda_{j}\right) T f\right)(y) \\
& +\sum_{j=1}^{l-1} \sum_{\sigma \in C_{j}^{l}} C_{j, l} A_{t_{B}}^{\prime}\left((b-\lambda)_{\sigma} T_{\vec{b}_{\sigma^{\prime}}} f\right)(y) \\
& +(-1)^{l} A_{t_{B}}^{\prime}\left(T\left(\prod_{j=1}^{l}\left(b_{j}-\lambda_{j}\right) f_{1}\right)\right)(y) \\
& +(-1)^{l} A_{t_{B}}^{\prime}\left(T\left(\prod_{j=1}^{l}\left(b_{j}-\lambda_{j}\right) f_{2}\right)\right)(y)
\end{aligned}
$$

Enhance,

$$
\begin{aligned}
& \frac{1}{\mu(B)} \int_{B}\left\|T_{\vec{b}} f(y)-A_{t_{B}}^{\prime}\left(T_{\vec{b}} f\right)(y)\right\|_{F} d \mu(y) \\
& \leq \frac{1}{\mu(B)} \int_{B}\left\|\prod_{j=1}^{l}\left(b_{j}(y)-\left(b_{j}\right)_{B}\right) T f(y)\right\|_{F} d \mu(y) \\
& +\frac{1}{\mu(B)} \int_{B}\left\|\sum_{j=1}^{l-1} \sum_{\sigma \in C_{j}^{l}} C_{j, l}\left(b(y)-b_{B}\right)_{\sigma} T_{\vec{b}_{\sigma^{\prime}}} f(y)\right\|_{F} d \mu(y) \\
& +\frac{1}{\mu(B)} \int_{B}\left\|T\left(\prod_{j=1}^{l}\left(b_{j}-\left(b_{j}\right)_{B}\right) f_{1}\right)(y)\right\|_{F} d \mu(y) \\
& +\frac{1}{\mu(B)} \int_{B}\left\|A_{t_{B}}^{\prime}\left(\prod_{j=1}^{l}\left(b_{j}-\left(b_{j}\right)_{B}\right) T f\right)(y)\right\|_{F} d \mu(y) \\
& +\frac{1}{\mu(B)} \int_{B}\left\|\sum_{j=1}^{l-1} \sum_{\sigma \in C_{j}^{l}} C_{j, l} A_{t_{B}}^{\prime}\left(\left(b-b_{B}\right)_{\sigma} T_{\vec{b}_{\sigma^{\prime}}} f\right)(y)\right\|_{F} d \mu(y) \\
& +\frac{1}{\mu(B)} \int_{B}\left\|A_{t_{B}}^{\prime}\left(T\left(\prod_{j=1}^{l}\left(b_{j}-\left(b_{j}\right)_{B}\right) f_{1}\right)\right)(y)\right\|_{F} d \mu(y) \\
& +\frac{1}{\mu(B)} \int_{B}\left\|T\left(\prod_{j=1}^{l}\left(b_{j}-\left(b_{j}\right)_{B}\right) f_{2}\right)(y)-A_{t_{B}}^{\prime}\left(T\left(\prod_{j=1}^{l}\left(b_{j}-\left(b_{j}\right)_{B}\right) f_{2}\right)\right)(y)\right\|_{F} d \mu(y) \\
& :=I+I I+I I I+I V+V+V I+V I I .
\end{aligned}
$$

Let us now estimate $I, I I, I I I, I V, V, V I$ and $V I I$ respectively. 
Take $1<\tau<p$ and $1<\tau^{\prime}<\infty$ such that $1 / \tau+1 / \tau^{\prime}=1$, then by Hölder's inequality and Lemma 4.2.2,

$$
\begin{aligned}
I & \leq\left(\frac{1}{\mu(B)} \int_{B}\left|\prod_{j=1}^{l}\left(b_{j}(y)-\left(b_{j}\right)_{B}\right)\right|^{\tau^{\prime}} d \mu(y)\right)^{1 / \tau^{\prime}}\left(\frac{1}{\mu(B)} \int_{B}\|T f(y)\|_{F}^{\tau} d \mu(y)\right)^{1 / \tau} \\
& \leq C\|\vec{b}\|_{*} M_{\tau}\left(\|T f\|_{F}\right)(x) .
\end{aligned}
$$

It is similar to the estimate of $I$,

$$
I I \leq C \sum_{j=1}^{l-1} \sum_{\sigma \in C_{j}^{l}} C_{j, l}\left\|\vec{b}_{\sigma}\right\|_{*} M_{\tau}\left(\left\|T_{\vec{b}_{\sigma^{\prime}}} f\right\|_{F}\right)(x) .
$$

For III, take $1<\gamma, \xi<\infty$ such that $1<\gamma \xi<p$. Then by Hölder's inequality, the boundedness of $T$ and Lemma 4.2.2, we have

$$
\begin{aligned}
I I I \leq & \left(\frac{1}{\mu(B)} \int_{B}\left\|T\left(\prod_{j=1}^{l}\left(b_{j}-\left(b_{j}\right)_{B}\right) f_{1}\right)(y)\right\|_{F}^{\gamma} d \mu(y)\right)^{1 / \gamma} \\
\leq & \frac{C}{\mu(B)^{1 / \gamma}}\left(\int_{2 B}\left\|\prod_{j=1}^{l}\left(b_{j}(y)-\left(b_{j}\right)_{B}\right) f(y)\right\|_{E}^{\gamma} d \mu(y)\right)^{1 / \gamma} \\
\leq & C\left(\frac{1}{\mu(B)} \int_{2 B}\left|\prod_{j=1}^{l}\left(b_{j}(y)-\left(b_{j}\right)_{B}\right)\right|^{\gamma \xi^{\prime}} d \mu(y)\right)^{1 / \gamma \xi^{\prime}} \\
& \times\left(\frac{1}{\mu(B)} \int_{2 B}\|f(y)\|_{E}^{\gamma \xi} d \mu(y)\right)^{1 / \gamma \xi} \\
\leq & C\|\vec{b}\|_{*} M_{\gamma \xi}\left(\|f\|_{E}\right)(x),
\end{aligned}
$$

here $1 / \gamma+1 / \gamma^{\prime}=1$

Take $\tau$ as in (4.2.6), then by Lemma 4.2.3,

$$
I V \leq C\|\vec{b}\|_{*} M_{\tau}\left(\|T f\|_{F}\right)(x)
$$

and

$$
V \leq C \sum_{j=1}^{l-1} \sum_{\sigma \in C_{j}^{l}} C_{j, l}\left\|\vec{b}_{\sigma}\right\|_{*} M_{\tau}\left(\left\|T_{\vec{b}_{\sigma^{\prime}}} f\right\|_{F}\right)(x) .
$$


For $V I$ we have

$$
\begin{aligned}
V I= & \frac{1}{\mu(B)} \int_{B} \| \int_{\mathbb{X}} a_{t_{B}}^{\prime}(y, z)\left(T\left(\prod_{j=1}^{l}\left(b_{j}-\left(b_{j}\right)_{B}\right) f_{1}\right)(z) d \mu(z) \|_{F} d \mu(y)\right. \\
\leq & \frac{1}{\mu(B)} \int_{B} \int_{2 B}\left\|a_{t_{B}}^{\prime}(y, z)\right\|_{\mathcal{L}(E, F)}\left\|T\left(\prod_{j=1}^{l}\left(b_{j}-\left(b_{j}\right)_{B}\right) f_{1}\right)(z)\right\|_{F} d \mu(z) d \mu(y) \\
& +\frac{1}{\mu(B)} \int_{B} \int_{\mathbb{X} \backslash 2 B}\left\|a_{t_{B}}^{\prime}(y, z)\right\|_{\mathcal{L}(E, F)}\left\|T\left(\prod_{j=1}^{l}\left(b_{j}-\left(b_{j}\right)_{B}\right) f_{1}\right)(z)\right\|_{F} d \mu(z) d \mu(y) \\
\leq & \frac{1}{\mu(B)} \int_{B} \int_{2 B} h_{t_{B}}(y, z)\left\|T\left(\prod_{j=1}^{l}\left(b_{j}-\left(b_{j}\right)_{B}\right) f_{1}\right)(z)\right\|_{F} d \mu(z) d \mu(y) \\
& +\frac{1}{\mu(B)} \int_{B} \int_{\mathbb{X} \backslash 2 B} h_{t_{B}}(y, z)\left\|T\left(\prod_{j=1}^{l}\left(b_{j}-\left(b_{j}\right)_{B}\right) f_{1}\right)(z)\right\|_{F} d \mu(z) d \mu(y) \\
:= & V I_{1}+V I_{2} .
\end{aligned}
$$

Thus by (4.2.1),

$$
\begin{aligned}
V I_{1} & \leq C \frac{1}{\mu(2 B) \mu(B)} \int_{B} \int_{2 B}\left\|T\left(\prod_{j=1}^{l}\left(b_{j}-\left(b_{j}\right)_{B}\right) f_{1}\right)(z)\right\|_{F} d \mu(z) d \mu(y) \\
& \leq C \frac{1}{\mu(2 B)} \int_{2 B}\left\|T\left(\prod_{j=1}^{l}\left(b_{j}-\left(b_{j}\right)_{B}\right) f_{1}\right)(z)\right\|_{F} d \mu(z) .
\end{aligned}
$$

So, by the estimate of $I I I$,

$$
V I_{1} \leq C\|\vec{b}\|_{*} M_{\gamma \xi}\left(\|f\|_{E}\right)(x),
$$

where $\gamma, \xi$ are as in (4.2.7).

For $V I_{2}$, by (4.2.3) and (1.5) we have

$$
\begin{aligned}
& V I_{2}=\frac{1}{\mu(B)} \int_{B} \sum_{k=1}^{\infty} \int_{2^{k+1} B \backslash 2^{k} B} h_{t_{B}}(y, z)\left\|T\left(\prod_{j=1}^{l}\left(b_{j}-\left(b_{j}\right)_{B}\right) f_{1}\right)(z)\right\|_{F} d \mu(z) d \mu(y) \\
& \leq \int_{B} \sum_{k=1}^{\infty} C \frac{2^{(k+1) n} s\left(2^{(k-1) m}\right)}{\mu(B) \mu\left(2^{k+1} B\right)} \int_{2^{k+1} B}\left\|T\left(\prod_{j=1}^{l}\left(b_{j}-\left(b_{j}\right)_{B}\right) f_{1}\right)(z)\right\| d \mu(z) d \mu(y) \\
& \leq \sum_{k=1}^{\infty} C \frac{2^{(k+1) n} s\left(2^{(k-1) m}\right)}{\mu\left(2^{k+1} B\right)} \int_{2^{k+1} B}\left\|T\left(\prod_{j=1}^{l}\left(b_{j}-\left(b_{j}\right)_{B}\right) f_{1}\right)(z)\right\|_{F} d \mu(z) .
\end{aligned}
$$


Take $\gamma, \xi$ as in the estimate of $I I I$, then by Hölder's inequality, Lemma 4.2.2 and (1.6), it is similar to the estimate of $I I I$,

$$
\begin{aligned}
& V I_{2} \leq \sum_{k=1}^{\infty} C \frac{2^{(k+1) n} s\left(2^{(k-1) m}\right)}{\mu\left(2^{k+1} B\right)^{1 / \gamma}}\left(\int_{2^{k+1} B}\left\|T\left(\prod_{j=1}^{l}\left(b_{j}-\left(b_{j}\right)_{B}\right) f_{1}\right)(z)\right\|_{F}^{\gamma} d \mu(z)\right)^{1 / \gamma} \\
& \leq \sum_{k=1}^{\infty} C \frac{2^{(k+1) n} s\left(2^{(k-1) m}\right)}{\mu\left(2^{k+1} B\right)^{1 / \gamma}}\left(\int_{2 B}\left\|\prod_{j=1}^{l}\left(b_{j}(z)-\left(b_{j}\right)_{B}\right) f(z)\right\|_{E}^{\gamma} d \mu(z)\right)^{1 / \gamma} \\
& \leq \sum_{k=1}^{\infty} 2^{k n} s\left(2^{(k-1) m}\right) 2^{-k n / \gamma}\left(\frac{1}{\mu(2 B)} \int_{2 B}\left|\prod_{j=1}^{l}\left(b_{j}(z)-\left(b_{j}\right)_{B}\right)\right|^{\gamma \xi^{\prime}} d \mu(z)\right)^{1 / \gamma \xi^{\prime}} \\
& \quad \times\left(\frac{1}{\mu(2 B)} \int_{2 B}\|f(z)\|_{E}^{\gamma \xi} d \mu(z)\right)^{1 / \gamma \xi} \\
& \leq \sum_{k=1}^{\infty} C 2^{k n} s\left(2^{(k-1) m}\right) 2^{-k n / \gamma}\|\vec{b}\|_{*} M_{\gamma \xi}\left(\|f\|_{E}\right)(x) \\
& \leq C\|\vec{b}\|_{*} M_{\gamma \xi}\left(\|f\|_{E}\right)(x) .
\end{aligned}
$$

Combining the estimates of $V I_{1}$ with $V I_{2}$, we get

$$
V I \leq C\|\vec{b}\|_{*} M_{\gamma \xi}\left(\|f\|_{E}\right)(x) .
$$

In the last, let us estimate $V I I$. Take $\tau$ and $\tau^{\prime}$ as in (4.2.6), then by Lemma 4.2.1 and Lemma 4.2.2, we have

$$
\begin{aligned}
V I I \leq & \frac{1}{\mu(B)} \int_{B}\left\|\left(T-A_{t_{B}}^{\prime} T\right)\left(\prod_{j=1}^{l}\left(b_{j}-\left(b_{j}\right)_{B}\right) f_{2}\right)(y)\right\|_{F} d \mu(y) \\
\leq & \frac{1}{\mu(B)} \int_{B} \int_{\mathbb{X} \backslash 2 B}\left\|K(y, z)-K_{t_{B}}(y, z)\right\|_{\mathcal{L}(E, F)} \\
& \times\left\|\prod_{j=1}^{l}\left(b_{j}(z)-\left(b_{j}\right)_{B}\right) f(z)\right\|_{E} d \mu(z) d \mu(y) \\
\leq & \frac{C}{\mu(B)} \int_{B} \sum_{k=1}^{\infty} \int_{2^{k+1} B \backslash 2^{k} B} \frac{1}{\mu(B(x, d(x, z)))} \frac{t_{B}^{\delta / m}}{d(y, z)^{\delta}} \\
& \times\left\|\prod_{j=1}^{l}\left(b_{j}(z)-\left(b_{j}\right)_{B}\right) f(z)\right\|_{E} d \mu(z) d \mu(y) \\
\leq & \sum_{k=1}^{\infty} C 2^{-k \delta} \frac{1}{\mu\left(2^{k+1} B\right)} \int_{2^{k+1} B}\left\|\prod_{j=1}^{l}\left(b_{j}(z)-\left(b_{j}\right)_{B}\right) f(z)\right\|_{E} d \mu(z) \\
\leq & \sum_{k=1}^{\infty} C 2^{-k \delta}\left(\frac{1}{\mu\left(2^{k+1} B\right)} \int_{2^{k+1} B}\left|\prod_{j=1}^{l}\left(b_{j}(z)-\left(b_{j}\right)_{B}\right)\right|^{\tau^{\prime}}\right)^{1 / \tau^{\prime}} d \mu(z) \\
& \times\left(\frac{1}{\mu\left(2^{k+1} B\right)} \int_{2^{k+1} B}\|f(z)\|_{E}^{\tau} d \mu(z)\right)^{1 / \tau} \\
\leq & C\|\vec{b}\|_{*} M_{\tau}\left(\|f\|_{E}\right)(x) .
\end{aligned}
$$


In fact, in the above estimate, we have used (1.3).

Combining the estimates of $I, I I, I I I, I V, V, V I$ with $V I I$, we get that for any $B \ni x$

$$
\begin{aligned}
& \frac{1}{\mu(B)} \int_{B}\left\|T_{\vec{b}} f(y)-A_{t_{B}}^{\prime}\left(T_{\vec{b}} f\right)(y)\right\|_{F} d \mu(y) \\
& \leq C\left(\|\vec{b}\|_{*} M_{\tau}\left(\|T f\|_{F}\right)(x)+\|\vec{b}\|_{*} M_{\gamma \xi}\left(\|f\|_{E}\right)(x)\right. \\
& \left.\quad+\sum_{j=1}^{l-1} \sum_{\sigma \in C_{j}^{l}} C_{j, l}\left\|\vec{b}_{\sigma}\right\|_{*} M_{\tau}\left(\left\|T_{b_{\sigma^{\prime}}} f\right\|_{F}\right)(x)\right) .
\end{aligned}
$$

Take supremum for all $B \ni x$, then we obtain (4.2.4).

From the above estimate for $M_{A^{\prime}}^{\#}\left(\left\|T_{\vec{b}} f\right\|_{F}\right)(x)$, it is easy to see that when $m=1$,

$$
\begin{aligned}
& \frac{1}{\mu(B)} \int_{B}\left\|T_{b_{1}} f(y)-A_{t_{B}}^{\prime}\left(T_{b_{1}} f\right)(y)\right\|_{F} d \mu(y) \\
& \leq C\left\|b_{1}\right\|_{*} M_{\tau}\left(\|T f\|_{F}\right)(x)+C\|\vec{b}\|_{*} M_{\gamma \xi}\left(\|f\|_{E}\right)(x) .
\end{aligned}
$$

Take supremum for all $B \ni x$ in (4.2.8) and use Lemma 2.2.4, we get that $T_{b_{1}}$ is bounded from $L_{E}^{p}(\mathbb{X})$ to $L_{F}^{p}(\mathbb{X})$. Enhance by induction on $l$ and (4.2.4), we finish the proof of Theorem 4.1.4.

4.3. The proofs of Theorem 4.1.5 and Theorem 4.1.6. Similar to the proof of Theorem 4.1.4, we need the following Lemmas.

Lemma 4.3.1([15]) For $0<\gamma$ and $0<\beta<n$, let

$$
M_{\beta, \gamma}(f)(x)=\sup _{B \ni x}\left(\frac{1}{\mu(B)^{1-\beta \gamma / n}} \int_{B}|f(y)|^{\gamma} d \mu(y)\right)^{1 / \gamma} .
$$

If $1<\gamma<q, 1<p<n / \beta$, such that $\beta \gamma / n<1$ and $1 / q=1 / p-\beta / n$, then there exists a constant $C>0$ such that

$$
\left\|M_{\beta, \gamma}(f)\right\|_{L^{q}} \leq C\|f\|_{L^{p}} .
$$

Lemma 4.3.2. Suppose that $(\mathbb{X}, d, \mu)$ is normal. Then for $0<\beta<1$ and $1 \leq q<\infty$, we have

$$
\sup _{B} \frac{1}{\mu(B)^{\beta / n}}\left(\frac{1}{\mu(B)} \int_{B}\left|f-f_{B}\right|^{q}\right)^{1 / q} \leq C\|f\|_{L i p(\beta)} .
$$

For $q=\infty$, the formula should be modified appropriately.

Lemma 4.3.3 Suppose that $(\mathbb{X}, d, \mu)$ is normal. Let $0<\beta_{1}, \beta_{2}, \ldots, \beta_{l}<1$ and $b_{j} \in \operatorname{Lip}\left(\beta_{j}\right)(j=1,2, \ldots, l)$. Then for any $1 \leq \gamma<\infty$ and $\sigma \in C_{j}^{l}(j=1,2, \ldots, l)$, there is a constant $C>0$ such that 


$$
\left(\frac{1}{\mu(B)} \int_{B}\left|\left(b(y)-b_{B}\right)_{\sigma}\right|^{\gamma} d \mu(y)\right)^{1 / \gamma} \leq C\left\|\vec{b}_{\sigma}\right\|_{L i p\left(\beta_{\sigma}\right)} \mu(B)^{\beta_{\sigma} / n} .
$$

Lemma 4.3.4 Suppose that $(\mathbb{X}, d, \mu)$ is normal. Let $H$ be a Banach space and $0<\beta_{j}<1$ for all $1 \leq j \leq l$. Assume that $\left\{A_{t}: t>0\right\}$ is an "approximation to the identity" on $H$ and $b_{j} \in \operatorname{Lip}\left(\beta_{j}\right)(j=1,2, \ldots, l)$. Then for every $f \in L_{H}^{p}(1<$ $p<\infty), \sigma \in C_{j}^{l}(j=1,2, \ldots, l)$ and $1<\gamma<\infty$, there is a constant $C>0$ such that

$$
\sup _{B \ni x} \frac{1}{\mu(B)} \int_{B}\left\|A_{t_{B}}\left(\left(b-b_{B}\right)_{\sigma} f\right)(y)\right\|_{H} d \mu(y) \leq C\left\|\vec{b}_{\sigma}\right\|_{L i p\left(\beta_{\sigma}\right)} M_{\beta, \gamma}\left(\|f\|_{H}\right)(x),
$$

where $t_{B}=r_{B}^{m}$ and $r_{B}$ is the radius of $\mathrm{B}$.

The method of proving Lemma 4.3.3 and Lemma 4.3.4 is analogous to that of proving Lemma 4.2.2 and Lemma 4.2.3. We omit it for brevity.

Let us now turn to prove Theorem 4.1.5. For any $x \in \mathbb{X}$, take $B=B\left(x_{0}, r_{B}\right) \ni$ $x$. Fix $f \in L_{E}^{p}(\mathbb{X})$, we set $f_{1}=f \chi_{2 B}$ and $f_{2}=f-f_{1}$. And denote $\vec{\lambda}=$ $\left(\lambda_{1}, \lambda_{2}, \ldots, \lambda_{l}\right)=\left(\left(b_{1}\right)_{B},\left(b_{2}\right)_{B}, \ldots,\left(b_{l}\right)_{B}\right)=\vec{b}_{B}$, then it is similar to (4.2.5),

$$
\frac{1}{\mu(B)} \int_{B}\left\|T_{\vec{b}} f(y)-A_{t_{B}}^{\prime}\left(T_{\vec{b}} f\right)(y)\right\|_{F} d \mu(y) \leq J_{1}+J_{2}+J_{3}+J_{4}+J_{5}+J_{6}+J_{7},
$$

where

$$
\begin{aligned}
& J_{1}=\frac{1}{\mu(B)} \int_{B}\left\|\prod_{j=1}^{l}\left(b_{j}(y)-\left(b_{j}\right)_{B}\right) T f(y)\right\|_{F} d \mu(y), \\
& J_{2}=\frac{1}{\mu(B)} \int_{B}\left\|\sum_{j=1}^{l-1} \sum_{\sigma \in C_{j}^{l}} C_{j, l}\left(b(y)-b_{B}\right)_{\sigma} T_{\vec{b}_{\sigma^{\prime}}} f(y)\right\|_{F} d \mu(y), \\
& J_{3}=\frac{1}{\mu(B)} \int_{B}\left\|T\left(\prod_{j=1}^{l}\left(b_{j}-\left(b_{j}\right)_{B}\right) f_{1}\right)(y)\right\|_{F} d \mu(y), \\
& J_{4}=\frac{1}{\mu(B)} \int_{B}\left\|A_{t_{B}}^{\prime}\left(\prod_{j=1}^{l}\left(b_{j}-\left(b_{j}\right)_{B}\right) T f\right)(y)\right\|_{F} d \mu(y), \\
& J_{5}=\frac{1}{\mu(B)} \int_{B}\left\|\sum_{j=1}^{l-1} \sum_{\sigma \in C_{j}^{l}}^{l} C_{j, l} A_{t_{B}}^{\prime}\left(\left(b-b_{B}\right)_{\sigma} T_{\vec{b}_{\sigma^{\prime}}} f\right)(y)\right\|_{F} d \mu(y), \\
& J_{6}=\frac{1}{\mu(B)} \int_{B}\left\|A_{t_{B}}^{\prime}\left(T\left(\prod_{j=1}^{l}\left(b_{j}-\left(b_{j}\right)_{B}\right) f_{1}\right)\right)(y)\right\|_{F} d \mu(y), \\
& J_{7}=\frac{1}{\mu(B)} \int_{B}\left\|T\left(\prod_{j=1}^{l}\left(b_{j}-\left(b_{j}\right)_{B}\right) f_{2}\right)(y)-A_{t_{B}}^{\prime}\left(T\left(\prod_{j=1}^{l}\left(b_{j}-\left(b_{j}\right)_{B}\right) f_{2}\right)\right)(y)\right\|_{F} d \mu(y) .
\end{aligned}
$$


Take $1<\tau<q$ such that $\beta \tau / n<1$. It is similar to the estimate of $I, I I, I V$ and $V$, by Hölder's inequality, Lemma 4.3.2, Lemma 4.3.3 and Lemma 4.3.4,

$$
J_{1}, J_{4} \leq C\|\vec{b}\|_{L i p(\beta)} M_{\beta, \tau}\left(\|T f\|_{F}\right)(x),
$$

and

$$
J_{2}, J_{5} \leq C \sum_{j=1}^{l-1} \sum_{\sigma \in C_{j}^{l}} C_{j, l}\left\|\vec{b}_{\sigma}\right\|_{L i p\left(\beta_{\sigma}\right)} M_{\beta, \tau}\left(\left\|T_{\vec{b}_{\sigma^{\prime}}} f\right\|_{F}\right)(x) .
$$

Take $1<\gamma, \xi<\infty$ such that $1<\gamma \xi<q$ and $\gamma \xi \beta / n<1$. Then it is similar to the estimates of $I I I$ and $V I$, by Lemma 4.3 .2 and Lemma 4.3.3,

$$
J_{3}, J_{6} \leq C\|\vec{b}\|_{L i p(\beta)} M_{\beta, \gamma \xi}\left(\|f\|_{E}\right)(x) .
$$

At last, using the kernel condition of $T$ and Lemma 4.3.2, it analogous to VII,

$$
J_{7} \leq C\|\vec{b}\|_{L i p(\beta)} M_{\beta, \tau}\left(\|T f\|_{F}\right)(x) .
$$

Thus we have

$$
\begin{aligned}
& \frac{1}{\mu(B)} \int_{B}\left\|T_{\vec{b}} f(y)-A_{t_{B}}^{\prime}\left(T_{\vec{b}} f\right)(y)\right\|_{F} d \mu(y) \\
& \leq C\left\{\|\vec{b}\|_{L i p(\beta)} M_{\beta, \tau}\left(\|T f\|_{F}\right)(x)+\sum_{j=1}^{l-1} \sum_{\sigma \in C_{j}^{l}} C_{j, l}\left\|\vec{b}_{\sigma}\right\|_{L i p\left(\beta_{\sigma}\right)} M_{\beta, \tau}\left(\left\|T_{\vec{b}_{\sigma^{\prime}}} f\right\|_{F}\right)(x)\right. \\
& \left.\quad+\|\vec{b}\|_{L i p(\beta)} M_{\beta, \gamma \xi}\left(\|T f\|_{F}\right)(x)\right\} .
\end{aligned}
$$

Take supremum for all $B \ni x$, it follows that

$$
\begin{aligned}
M_{A^{\prime}}^{\#}\left(\left\|T_{\vec{b}} f\right\|_{F}\right)(x) \leq & C\left\{\|\vec{b}\|_{L i p(\beta)} M_{\beta, \tau}\left(\|T f\|_{F}\right)(x)+\|\vec{b}\|_{L i p(\beta)} M_{\beta, \gamma \xi}\left(\|T f\|_{F}\right)(x)\right. \\
& \left.+\sum_{j=1}^{l-1} \sum_{\sigma \in C_{j}^{l}} C_{j, l}\left\|\vec{b}_{\sigma}\right\|_{L i p\left(\beta_{\sigma}\right)} M_{\beta, \tau}\left(\left\|T_{\vec{b}_{\sigma^{\prime}}} f\right\|_{F}\right)(x)\right\} .
\end{aligned}
$$

So, discussing as in the proof of Theorem 4.1.4, by induction on $l$ and Lemma 4.2.4 and Lemma 4.3.1 we get the desired results.

In the following we will prove Theorem 4.1.6.

It is enough to show that for any ball $B$,

$$
\frac{1}{\mu(B)^{1+\beta / n-1 / p}} \int_{B}\left\|T_{b} f(x)-A_{t_{B}}^{\prime}(T f)(x)\right\|_{F} d \mu(x) \leq C\|b\|_{L i p(\beta)}\|f\|_{L_{E}^{p}(\mathbb{X})} .
$$


For $f \in L_{E}^{p}(1<p<\infty)$, set $B=B\left(x_{0}, r_{B}\right), f_{1}=f \chi_{2 B}$ and $f_{2}=f-f_{1}$, then $\frac{1}{\mu(B)^{1+\beta / n-1 / p}} \int_{B}\left\|T_{b} f(x)-A_{t_{B}}^{\prime}\left(T_{b} f\right)(x)\right\|_{F} d \mu(x)$ $\leq \frac{1}{\mu(B)^{1+\beta / n-1 / p}} \int_{B}\left\|T_{b} f_{1}(x)\right\|_{F} d \mu(x)+\frac{1}{\mu(B)^{1+\beta / n-1 / p}} \int_{B}\left\|A_{t_{B}}^{\prime}\left(T_{b} f_{1}\right)(x)\right\|_{F} d \mu(x)$ $+\frac{1}{\mu(B)^{1+\beta / n-1 / p}} \int_{B}\left\|T_{b} f_{2}(x)-A_{t_{B}}^{\prime}\left(T_{b} f_{2}\right)(x)\right\|_{F} d \mu(x)$ $:=U_{1}+U_{2}+U_{3}$,

where $t_{B}=r_{B}^{m}$.

Choose $p_{1}$ satisfying $1<p_{1}<n / \beta \leq p$ and take $q_{1}$ such that $1 / q_{1}=1 / p_{1}-\beta / n$, then by Hölder's inequality and the boundedness of $T_{b}$ (see Theorem 4.1.5),

$$
\begin{aligned}
U_{1} & \leq \frac{1}{\mu(B)^{1+\beta / n-1 / p}}\left(\int_{B}\left\|T_{b} f_{1}(x)\right\|_{F}^{q_{1}} d \mu(x)\right)^{1 / q_{1}} \mu(B)^{1-1 / q_{1}} \\
& \leq \frac{C\|b\|_{L i p(\beta)}}{\mu(B)^{\beta / n-1 / p+1 / q_{1}}}\left(\int_{2 B}\|f(x)\|_{E}^{p_{1}} d \mu(x)\right)^{1 / p_{1}} \\
& \leq \frac{C\|b\|_{L i p(\beta)}}{\mu(B)^{\beta / n-1 / p+1 / q_{1}}} \mu(B)^{1 / p_{1}-1 / p}\|f\|_{L_{E}^{p}(\mathbb{X})} \\
& \leq C\|b\|_{L i p(\beta)}\|f\|_{L_{E}^{p}(\mathbb{X})}
\end{aligned}
$$

For $U_{2}$, we have

$$
\begin{aligned}
U_{2}= & \frac{1}{\mu(B)^{1+\beta / n-1 / p}} \int_{B} \int_{2 B} h_{t_{B}}(x, y)\left\|T_{b} f_{1}(y)\right\|_{F} d \mu(y) d \mu(x) \\
& +\frac{1}{\mu(B)^{1+\beta / n-1 / p}} \int_{B} \sum_{k=1}^{\infty} \int_{2^{k+1} B \backslash 2^{k} B} h_{t_{B}}(x, y)\left\|T_{b} f_{1}(y)\right\|_{F} d \mu(y) d \mu(x) \\
:= & U_{21}+U_{22} .
\end{aligned}
$$

Since for any $x \in B$ and $y \in 2 B$, by (1.5) we have

$$
h_{t_{B}}(x, y) \leq C \mu(B)^{-1} \text {. }
$$

Then

$$
U_{21} \leq \frac{C}{\mu(B)^{1+\beta / n-1 / p}} \int_{2 B}\left\|T_{b} f_{1}(y)\right\|_{F} d \mu(y) .
$$

Thus by the estimate of $U_{1}$,

$$
U_{21} \leq C\|b\|_{L i p(\beta)}\|f\|_{L_{E}^{p}(\mathbb{X})} .
$$

Moreover, for any $x \in B$ and $y \in 2^{k+1} B \backslash 2^{k} B$, we have $d(x, y) \geq 2^{k-1} r_{B}$ and $h_{t_{B}}(x, y) \leq C \frac{s\left(2^{(k-1) m}\right)}{\mu(B)}$. 
So take $p_{1}$ and $q_{1}$ as in (4.3.1), then it is similar to the estimate (4.3.1),

$$
\begin{aligned}
U_{22} & \leq C \sum_{k=1}^{\infty} \frac{s\left(2^{(k-1) m}\right)}{\mu(B)^{1+\beta / n-1 / p}} \int_{2^{k+1} B}\left\|T_{b} f_{1}(y)\right\|_{F} d \mu(y) \\
& \leq C \sum_{k=1}^{\infty} \frac{s\left(2^{(k-1) m}\right)}{\mu(B)^{1+\beta / n-1 / p}}\left(\int_{2^{k+1} B}\left\|T_{b} f_{1}(y)\right\|_{F}^{q_{1}} d \mu(y)\right)^{1 / q_{1}} \mu\left(2^{k+1} B\right)^{1-1 / q_{1}} \\
& \leq C\|b\|_{L i p(\beta)} \sum_{k=1}^{\infty} \frac{2^{k n\left(1-1 / q_{1}\right)} s\left(2^{(k-1) m}\right)}{\mu(B)^{\beta / n-1 / p+1 / q_{1}}}\left(\int_{2 B}\|f(y)\|_{E}^{p_{1}} d \mu(y)\right)^{1 / p_{1}} \\
& \leq C\|b\|_{L i p(\beta)} \sum_{k=1}^{\infty} \frac{2^{-k\left(n / q_{1}+N+\tau\right)} 2^{k(n+N+\tau)} s\left(2^{(k-1) m}\right)}{\mu(B)^{\beta / n+1 / q_{1}-1 / p_{1}}}\left(\int_{2 B}\|f(y)\|_{E}^{p} d \mu(y)\right)^{1 / p} \\
& \leq C\|b\|_{L i p(\beta)}\|f\|_{L_{E}^{p}(\mathbb{X}) .}
\end{aligned}
$$

Moreover, by $x \in B$, the definition of $b \in \operatorname{Lip}(\beta)$, Hölder's inequality and Lemma 4.3.2, we have

$$
\begin{aligned}
& \left\|T_{b} f_{2}(x)-A_{t_{B}}^{\prime}\left(T_{b} f_{2}\right)(x)\right\|_{F} \\
& \leq \int_{\mathbb{X} \backslash 2 B}^{\infty}\left\|K(x, y)-K_{t_{B}}(x, y)\right\|_{\mathcal{L}(E, F)}\|(b(x)-b(y)) f(y)\|_{E} d \mu(y) \\
& \leq C \sum_{k=1}^{\infty} \int_{2^{k+1} B \backslash 2^{k} B} \frac{1}{\mu(B(x, d(x, y)))} \frac{t_{B}^{\delta / m}}{d(x, y)^{\delta}}\|(b(x)-b(y)) f(y)\|_{E} d \mu(y) \\
& \leq C \sum_{k=1}^{\infty} 2^{-\delta k} \mu\left(2^{k+1} B\right)^{-1} \int_{2^{k+1} B}|b(x)-b(y)|\|f(y)\|_{E} d \mu(y) \\
& \leq C \sum_{k=1}^{\infty} 2^{-\delta k} \mu\left(2^{k+1} B\right)^{-1}\left(\int_{2^{k+1} B}|b(x)-b(y)|^{p^{\prime}} d \mu(y)\right)^{1 / p^{\prime}}\left(\int_{2^{k+1} B}\|f(y)\|_{E}^{p} d \mu(y)\right)^{1 / p} \\
& \leq C\|b\|_{L i p(\beta)} \sum_{k=1}^{\infty} \mu\left(2^{k+1} B\right)^{\beta / n-1 / p} 2^{-\delta k}\|f\|_{L_{E}^{p}(\mathbb{X})} \\
& \leq C \mu(B)^{\beta / n-1 / p}\|b\|_{L i p(\beta)} \sum_{k=1}^{\infty} 2^{-(\delta / n+1 / p-\beta / n) n k}\|f\|_{L_{E}^{p}(\mathbb{X})} \\
& \leq C \mu(B)^{\beta / n-1 / p}\|b\|_{L i p(\beta)}\|f\|_{L_{E}^{p}(\mathbb{X})}
\end{aligned}
$$

So,

$$
U_{3} \leq C\|b\|_{L i p(\beta)}\|f\|_{L_{E}^{p}\left(\mathbb{R}^{n}\right)} .
$$

Combining the estimates of $U_{1}, U_{2}$ with $U_{3}$ we complete the proof.

\section{REFERENCES}

[1] Coifman, R. R. and Weiss, G., Analyse harmonique non commutative sur certains espaces homogenènes, Lecture Notes in Math., 242, Springer, 1971. 
[2] Macías, R. A. and Segovia. C., Lipschitz function on spaces of homogeneous type, Adv. Math., 33(1979), 257-270.

[3] Macías, R. A. and Segovia. C., A decomposition into atoms of distribution on spaces of homogeneous type, Ibid., 271-309.

[4] Hörmander, L., Estimate for translation invariant operators on $L^{p}$ spaces, Acta math., 104(1960), 93-139

[5] Fefferman, C., Inequalities for strongly singular convolution operators. Acta Math., 124(1970), 9-36.

[6] Christ, M, Weak-type $(1,1)$ bounds for rough operators, Ann. Math. 128(1988), 19-42.

[7] Christ, M. and Rubio de Francia J., Weak-type $(1,1)$ bounds for rough operators II, Invent. Math., 93(1988), 225-237.

[8] Duong, X. T. and McIntosh. A., Singular integral operators with non-smooth kernels on irregular domains, Rev. Math. Iberoamericana., 15(1999), 233-265.

[9] Duong, X. T. and Yan. L., Commutator of BMO function and Singular integral operators with non-smooth kernels, Bull. Aus. Math. Soc., 67(2003), 187-200.

[10] Martell, J. M., Sharp maximal function assoiated with approximations of the identity in spaces of homogeneous type and applications, Studia Math., 161(2004), 113-145.

[11] Duong, X. T. and Yan. L., New function spaces of BMO type, the John-Nirenberg inequality, interpolation and applications, to appear in Comm. Pure Appl. Math., 2004.

[12] Duong, X. T. and Robinson. D., Semigroup kernels, Poisson bounds and holomorphic calculus, J. Funct. Anal., 142(1996), 89-128.

[13] Duoanikoetxea, J., Fourier Analysis, 29(2000), Grad. Stud. Math. 29, Amer. Math. Soc., Providence, RI, 2000.

[14] Chanillo, S., A note on commutators. Indiana Univ Math. 44(1982). 7-16

[15] Qiu, D., Some of integral operators on spaces of homogneous type, Ann. Math (Chinese)., $22(2001), 797-804$.

Hui-Xia Mo

School of Science

Beijing University of Post and Telecommunications

Beijing 100876, P. R. China

E-mail:huixmo@163.com

Shan-zhen Lu

School of Mathematical Sciences

Beijing Normal University

Beijing 100875, P. R. China

E-mail: lusz@bun.edu.cn 